\title{
1 Longevity interventions temporally scale healthspan in Caenorhabditis elegans
}

2 Cyril Statzer ${ }^{1 \S}$, Peter Reichert ${ }^{2 \S}$, Jürg Dual ${ }^{2 *}$, and Collin Y. Ewald ${ }^{1 *}$

3

41 Laboratory of Extracellular Matrix Regeneration, Institute of Translational Medicine,

5 Department of Health Sciences and Technology, ETH Zürich, Schwerzenbach CH-8603,

6 Switzerland

72 Eidgenössische Technische Hochschule Zürich, Department of Mechanical and Process

8 Engineering, Institute for Mechanical Systems, Zürich CH-8092, Switzerland

9

$10 \S$ Authors contributed equally

$11 *$ Corresponding authors: collin-ewald@ethz.ch (CYE) and dual@imes.mavt.ethz.ch (JD)

12

13 Keywords: acoustophoresis, microfluidics, healthy aging, maximum muscle strength,

14 healthspan, sickspan, aging biomarker, lifespan machine 


\section{Abstract}

Longer lived individuals, such as centenarians or longevity mutants of model organisms, have

later onsets and lower incidence rates of late-life morbidities or disabilities than the average

population. However, whether increased lifespan is caused by a compression of the portion of

life spent in a state of morbidity, i.e., "sickspan," is highly debated. It is unclear which health matrices are representative for measuring healthspan (time spent in good health); however, muscular performance is generally a good indicator for the health status in humans and a predictor for their mortality, regardless of the underlying cause. Here, we developed a novel microfluidic device that employs acoustophoretic force fields to quantify the maximum muscle strength and dynamic power in aging $C$. elegans populations. We found that longevity mutants have a delayed onset and lower declining rates in maximum muscle strength compared to wild type. Reconciling previous conflicting reports, we confirmed that certain longevity mutants exhibited a mild increase in relative sickspan measured by voluntary movement matrices, which is not the case when using our acoustophoretic force measurements, swim endurance, or other approaches. Using six different biomarkers for healthspan, we observed a time-dependent onset of morbidity, starting with a loss of stress resilience, a decline in dynamic power, and a decline in structural integrity, culminating finally in inactivity (lethargy) and a loss of mobility. We observed that a subset of aging biomarkers correlate with each other and maybe functionally interconnected. Surprisingly, we did not observe a compression of sickspan in longevity mutants but instead observed a temporal scaling of healthspan with diminishing returns for extreme lifespan extensions. Given the conservation of these longevity interventions, this raises the question of whether the healthspan of mammalian longevity interventions is temporally scaled as well. 


\section{Introduction}

The continuously growing elderly population is projected to result in 1.5 billion people above the age of 65 globally by $2050^{1}$. This poses a significant challenge since old age is the major risk factor for developing cancer, dementia, cardiovascular, and metabolic diseases ${ }^{2}$, especially since people suffer for approximately $20 \%$ of their lifespan from one or multiple of these chronic illnesses, which are themselves accompanied by other late-life disabilities ${ }^{2}$. Current estimates indicate that delaying the onset of these chronic diseases by two years would save $\$ 7.1$ trillion over the next fifty years in the US alone ${ }^{3}$. Therefore, major research efforts are dedicated to understanding how to increase the time spent in good health (i.e., healthspan) and to postpone and compress the time spent suffering from age-related pathologies and chronic diseases (i.e., sickspan) ${ }^{2,4-6}$.

People that are more than one hundred years old, so-called centenarians, display a delayed onset and a lower incidence rate of late-life morbidities compared to people in the age bracket of 80 to 89 years ${ }^{7-12}$. Genome-wide association studies have shown associations between the exceptional longevity of centenarians and aging-related genes identified in model organisms ${ }^{2,13,14}$. Mutations in genes that promote longevity in model organisms, such as $C$. elegans, have been instrumental in identifying mechanisms that promote healthy aging ${ }^{2,13-15}$.

A recent study has questioned this approach of using C. elegans longevity mutants to gain insights for promoting healthy aging or mechanisms that prolong healthspan ${ }^{16}$. Using four matrices (resilience to heat and oxidative stress, voluntary movement, and swimming performance) to assess the "health" status of aging C. elegans, they found that four commonly used longevity mutants outperformed wild type at any given timepoint at older ages, consistent with previous reports. However, compared to their maximum lifespan, longevity mutants displayed an increased sickspan-to-healthspan ratio compared to wild type ${ }^{16}$. Other studies have not observed an increase of sickspan in long-lived C. elegans mutants, except in the case of lower mobility or movement scores for the insulin/IGF-1 receptor longevity daf-2(e1370) 
67

mutants ${ }^{17-20}$. A large part of the "prolonged sickspan" based on the motility of these daf2(e1370) mutants was attributed to behavioral preferences of food over exploration linked to $o d r-10$ gene expression ${ }^{17}$ or improper dauer-like quiescence behavior ${ }^{20-24}$. Although all these studies showed that sickspan is not increased in longevity mutants, the question remained about how healthspan changes when the lifespan is extended. We hypothesized that using other health matrices independent of voluntary or behavioral influences, such as physical properties of muscular strength, which is one of the best predictors for all-cause mortality in humans ${ }^{25}$, we might be able to quantify the health trajectory of $C$. elegans longevity mutants.

Here we confirm that voluntary movement during aging declines and this fragility is not extended in longevity mutants, except mildly in daf-2 mutants, using high-resolution lifespan and movement measurements on plates. Instead of assessing swimming speed, we measured swimming endurance and found $d a f-2$ longevity mutants outperforming wild type during aging. We developed a novel microfluidic device and applied acoustophoretic force fields to quantify the maximum force and power of C. elegans. Using a high frequency and high power acoustic force field, it becomes possible to set up a contactless, constant in time, and uniform force field acting along the whole $C$. elegans body. Therefore, this force field challenges swimming $C$. elegans in a similar way body-weight exercises do for humans in a gravity field. Furthermore, the acoustic field showed to be suited for stimulating a swimming response of resting $C$. elegans. All longevity mutants showed delayed onset of the decline in maximum force and dynamic power during aging. We observed heterogeneity between individuals across all genotypes in the onset of age-related phenotypes, several correlated phenotypes, and a timedependent occurrence of multiple disabilities. However, we did not find a compression of sickspan, but rather a temporal scaling of healthspan relative to their maximal lifespan across genotypes. 


\section{Results}

\section{Voluntary movement healthspan is proportionally increased by longevity interventions}

To obtain highly quantitative data on lifespan and healthspan, we used a lifespan machine ${ }^{26}$. Here, we defined the "voluntary movement healthspan" as the time spent fast crawling and the "voluntary movement sickspan" as the time spent slow crawling or displaying minimal posture changes (see Materials and Methods for detailed definition). We chose eat-2(ad1116) as a genetic model for dietary restriction-mediated longevity, $g l p-1(e 2141)$ as a genetic model for germ-stem-cell-less-mediated longevity, daf-2(e1368) and daf-2(e1370) as genetic models for reduced insulin/IGF-1 signaling mediated longevity. We cultured all animals at the same temperature $\left(15^{\circ} \mathrm{C}\right)$ and in the same environment with the same food source, except $g l p-1$ that underwent a brief temperature upshift during development as in preparation for the lifespan assay. To avoid dauer-specific traits that occur in reduced insulin/IGF-1 signaling mutants ${ }^{22}$, and to avoid pathogenicity from a bacterial food source ${ }^{20}$, lifespans were run at $15^{\circ} \mathrm{C}$ on heatkilled bacteria. Thus, the experimental setup was designed to offer optimal conditions and was kept identical while C. elegans genotypes were varied.

As expected, we measured a significant increase in lifespan for these long-lived mutants compared to wild type (Fig. 1a; Supplementary Table 1, Supplementary Video 1). Interestingly, our data showed that the longer-lived the mutant was, the more prolonged was the voluntary movement healthspan (Fig. 1b). Therefore, interventions that increase lifespan also increase the time spent moving fast and actively.

\section{Relative increase for both health- and sickspan in long-lived mutants}

To better understand the rescaling of the time spent in frailty in these long-lived mutants, we analyzed the fraction of slow-moving animals per day. We observed gaussian activity distributions, which were shifted along the time axis for these longevity mutants (Fig. 1c). This delayed onset of the sickspan (Fig. 1c) is consistent with the prolonged healthspan of these 
long-lived mutants (Fig. 1b). However, except for dietary restricted eat-2 mutants, the width and the area of the Gaussian distributions were bigger for long-lived mutants than wild type

to wild type. Next, we asked whether the fraction spent in health- and sickspan during the

\section{Heterogeneity in the length of sickspan but a fixed onset of sickspan}

Since we did not observe a compression of voluntary movement sickspan of the entire

each genotype, we were surprised to measure such a vast heterogeneity (Fig. 1d), given that all

these individual animals of a population are genetically identical, consume the same food, and are housed in the same environment. Only the $g l p-1$-mediated longevity showed an overall compression of individual sickspan traces (Fig. 1d). For comparison among these different genotypes, we decided to use "relative age" by dividing lifespan curves into quartiles and computing the health-to-sickspan ratio for each quartile (Fig. 1d insets; Extended Data Fig. 2).

137 Consistent with previous reports on wild type ${ }^{27}$, we found that in the first quantile of the lifespan curve, individual animals spent about $90 \%$ of their lifetime fast-moving and $10 \%$ slowmoving, indicating that these animals die young with a compressed sickspan compared to the last quartile wherein animals spent about $75 \%$ of their lifetime fast-moving and $25 \%$ slow-

141 moving (Fig. 1d insets; Extended Data Fig. 1). Remarkably, it looks like the onset of an 142 individual's sickspan is a fixed event starting approximately when the first $10 \%$ of the isogenic 
143 population starts to die (Fig. 1d). This observation suggests that up to a certain time point, the 144 animal's physiological integrity is maintained. After this time point, there appears to be a 145 stochastic decay resulting in a heterogenous sickspan distribution. Viewing the data using this 146 alternative interpretation of a fixed onset of sickspan would explain why animals in the first 147 quartile of the lifespan curve die young and spend less time in poor health, while animals in the 148 last quartile of the lifespan curve die old and spend more time in poor health. Thus, the time spent fast- vs. slow-moving seems to have a fixed onset in time.

\section{Voluntary movement healthspan temporally scales with lifespan except in daf-2 mutants}

The model of a fixed onset-timepoint for frailty would suggest that longevity interventions would simply delay the onset. To address this, we contrasted the number of days spent fastmoving (healthspan) for each individual as a function of their time lived (lifespan in days; Fig. (Fig. 1e). Furthermore, the $g l p-1$ with an R squared of 0.8 and $d a f-2(e 1368)$ with an R squared of 0.5 indicate lower or higher heterogeneity, respectively, compared to wild type (Fig. 1e). This is also apparent in the increased or decreased spread of data points below the regression line in Fig. 1e and by increased or decreased lengths of the individual sickspan traces in Fig. $1 \mathrm{~d}$, respectively. One interesting aspect to note is that individuals in quartile 2 and 3 , which expire in the middle of the lifespan curves, displayed shortened healthspan relative to their lifespan, whereas individuals in the last quartile showed an extended healthspan relative to their lifespan (Fig. 1e). This might be because sicker individuals simply died earlier, leading to an enrichment of healthier-aging individuals in the last quartile (Extended Data Fig. 1, 2). Based on the high $\mathrm{R}$ squared values for all genotypes, we applied a linear model to investigate the relationship between health- and lifespan (Fig. 1e). Steeper linear regression lines compared to wild type would indicate an increase in health- to lifespan ratio. The slopes of the linear model 
169

170

171

172

173

174

175

176

177

178

179

180

181

182

were steeper for eat-2 and $g l p-1$, but less steep for the two daf-2 mutants compared to wild type (Fig. 1e), suggesting that $g l p-1$ and eat-2 spent a larger fraction and daf-2 mutants spent a smaller fraction of their lifespan actively moving. Since slopes of linear models can be sensitive to extreme values, we compared the population means of health- and lifespan across all genotypes (Fig. 1f). When we extrapolated the mean healthspan to mean lifespan ratio of wild type, we found that eat-2 and $g l p-1$ were close to this extrapolated line, whereas the daf-2 mutants lacked approximately three days (i.e., 7\%) of mean healthspan in respect to their mean relative lifespan (Fig. 1f). Thus, we uncovered that the prolonged voluntary movement healthspan temporally scales with the prolonged lifespan for each of these longevity mutants except less stringently for $d a f-2$ mutants.

\section{Exhaustion of Swimming endurance and stress resilience during aging}

Thus far, our observations and interpretations on healthspan are based on the decline of voluntary movement on culturing plates in the abundance of food. Certain genotypes like daf2(e1370) are less motivated to forage and display a more rapid decline in voluntary foraging behavior compared to wild type leading to the interpretation of being less healthy ${ }^{17}$. In our setting, this lower foraging behavior is less pronounced in daf-2(e1370) since they were cultured at $15^{\circ} \mathrm{C}$, an environment that leads to less dauer program activation ${ }^{22}$. We used a second approach to assess health based on voluntary movement and conducted a swimming assay in the absence of food. Placing C. elegans in a water-based physiological buffer (M9 buffer) stimulates active trashing. When animals were young, they swim for more than 30 hours without a decline in activity levels (Fig. 2). In the case of older animals, this endurance capacity declines but less rapidly in longevity mutants (Fig. 2). Similarly, exposing aging animals to the oxidative-stress-inducing agent arsenite, we report a progressive decline in stress resilience of wild-type animals (Fig. 2). This decline in stress resilience is shifted to chronological older animals in long-lived strains compared to wild type (Fig. 2). For both endurance swimming and 
195

196

197

198

stress resilience, aging $d a f-2$ mutants surpassed chronological age-matched wild-type animals (Fig. 2). Taken together, these results suggest that the observed deficit in healthspan of daf-2 mutants using a food-dependent movement assay might be largely due to their preferences in lower foraging or exploratory behavior ${ }^{17}$ rather than a loss of capability or frailty ${ }^{16}$. Moreover, the progressive decline in endurance swimming and stress resilience during aging occurs comparably in both wild type and longevity mutants but is delayed by just 1 or 2 days of chronological age for longevity mutants (Fig. 2). This difference between two voluntary movement assays led us to develop an inducible, motivation-independent exercise platform for C. elegans. Our goal is to address the following shortcomings of current methods: the movement should be inducible with a strong stimulus and not dependent on secondary cues like food or intrinsic motivation to thrash, it should be measurable in a short time window to assess health in this instant, and it should directly measure a physiologically relevant parameter like maximum muscle force or functional tissue integrity. This is especially important when comparing different genotypes, which often respond differently to their environment.

\section{Acoustophoretic characterization of $C$. elegans force and muscle power}

In humans, one of the best predictors for all-cause mortality is the decline in muscle maximum force and power ${ }^{25,28,29}$. However, a tool or device to quantify the maximum force and power of $C$. elegans muscles did not exist. The application potential would be immense since $C$. elegans muscle structures are strongly conserved, as in mammals, and forced maximum strength measurements to the point of collapse would be unethical in mammalian models. We developed a microfluidic device harnessing the power of acoustic standing waves (Fig. 3a, 3b, Supplementary Video 2, 3, Supplementary Methods). We have recently applied ultrasonic waves to compress, move and quantitatively characterize larval C. elegans ${ }^{30}$. We reasoned that we could employ ultrasonic standing waves to trap and stretch out C. elegans in the minima of the acoustic force fields (Fig. 3c, 3d). C. elegans dislike being trapped and try to escape by 
applying mechanical forces (body bending) against the acoustic force field (Fig. 3c, 3d). The further away from the acoustic force field minimum, the harder it gets to move against the force as omega reversals ${ }^{31}$. Thus, the degree of deflection of the C. elegans body away from the acoustic force field minimum provides an estimate of the maximal muscle strength the animal can master to try to escape the acoustic trap.

\section{Muscular strength declines in aging $C$. elegans}

To quantify muscular forces, we developed a model by dividing the C. elegans body plan into 13 rigid links connected by joints along the animal's midline (Fig. 3e). Upon applying acoustic force fields, we measured the deflection of these 13 nodes for 30 seconds and the number of times the animal escaped the force fields (Fig. 3f). A typical exercise round is structured in up to ten cycles consisting of 30 seconds of ultrasonic force and a 5-second break (Fig. 3g). We measured the muscular forces of aging wild-type C. elegans (Fig. 3g). After 3-5 cycles, we observed muscle fatigue, which set in earlier the older the animals were (Fig. 3g). We observed first an increase and then a decrease in the heterogeneity of individual C. elegans muscular strengths (Fig. 3g). Similarly, we first saw an increase and then a progressive decline in muscle terms of muscle strength and function at day 20 of adulthood (Fig. 3h). This indicates the

241 preservation of muscle power in aging longevity mutants.

The overall force and power of a $C$. elegans depend on its muscle strength as well as its total 
in body size beyond the reproductive period and then shrunk during aging (Fig. 4a, 4b). A prolonged growing phase correlates with longevity ${ }^{32}$. We found that longevity mutants prolonged their growing phase and shrunk less than wild-type animals during aging (Fig. 4a, 4b). Structural integrity declines during C. elegans aging, such as internal organ atrophy ${ }^{34}$, loss of internal pressure (Supplementary Method) ${ }^{35}$, and disorganization of the exoskeleton cuticle occurs ${ }^{36}$. We noticed that in the acoustic force field, C. elegans undergoes compression, and this compressibility stays fairly constant during aging (Fig. 4d, 4e). We conclude that although

young $C$. elegans can overcome the acoustic force field leading to an omega turn eight times each cycle (Fig. 4e, Supplementary Video 4). The ability to overcome the force field and turn in the microfluidic chip progressively declines during aging but is preserved in longevity mutants (Fig. 4e, Extended Data Fig. 3). Turning in the chip can be viewed as a measure of also showcases that the animal is not placid but trying to escape. Next, we assessed the overall energy per individual $C$. elegans as an assessment of overall body volume deflected against the force field. We found an increase of energy per individual until mid-age and then a decline (Fig. 4f) reminiscent of the longitudinal body size curve (Fig. 4a). In our measurements to determine the overall force and power of $C$. elegans, the body size is a confounding factor. In all our longitudinal measurements, we had included a positive control in the form of a muscle267 defective mutant (CB190) that carries a mutation in the muscle myosin class II heavy chain 268 (unc-54). These muscle-constriction-defective mutants were unable to perform omega turns in 269 the chip but showed similar compressibility and longitudinal growth curves, illustrating that the 270 rise and fall of the overall energy are confounded by the organismal growth curve. Therefore, 271 we decided to use the dynamic power as defined as energy expenditure relative to the previous 272 time point and normalized it by the volume of each animal (i.e., volume; Fig. 4g). Using a 
273 human analogy, total refers to how long a weight can be lifted, dynamic power only considers

274 the process of lifting the weight without holding the weight, and dynamic power takes the

275 weight of the person lifting the weight into account. In this way, we found that the overall force 276 and power of longevity mutants were preserved for almost three-quarters (55-83\%) compared 277 to about one-third (30\%) of their lifespan in wild type (Fig. 4g). Thus, muscular strength is 278 maintained longer in longevity mutants.

\section{Temporal scaling of age-related pathologies in longevity mutants}

281 Next, we asked whether other age-related pathologies or morphological changes show any 282 delayed onset on longevity mutants compared to wild type. We quantified 592 animals, 283 investigated timepoints between day 0 and day 33 (12 animals on average per strain and time point) at the first two cycles of actuation (1183-time sequences), which comprised over 800 '000 frames in total. We then manually quantified additional morphological changes such as intestine length and diameter, pixel intensity, wrinkles in the cuticle, and pharynx diameter in a subsampled representative subset (approx. 50’000 frames; Extended Data Fig. 4). Although not all, many age-related phenotypes were delayed in their onset and displayed a slowed decline in longevity mutants compared to wild type (Fig. 5, Extended Data Fig. 5). Rescaling phenotypic trajectories of wild type by the lifespan extension observed in the long-lived strains revealed that many closely match the trajectories observed in long-lived strains for both daf-2 mutants (Fig. 5C). Notably, for animals' length, diameter, volume, and intestine length, phenotypic 293 scaling was observed when comparing wild type to daf-2(e1368) and daf-2(e1370). In the case 294 of eat-2, the observed lifespan extension was too limited to draw conclusions and $g l p-1$ never ceased growing. The severely paralyzed myosin mutant unc-54(e190) displayed the opposite 
observed that the starting values as young adults are often similar (Fig. 5D). However, the slopes and point of decline are shifted compared to wild type. change similarly during aging, that many are temporally scaled in longevity interventions, and that maximum muscle strength offers an orthogonal perspective on studying aging compared to physiological features. This highlights the importance of performing high-intensity muscle strength measurements when studying physiological aging and quantifying healthspan.

\section{Longevity mutants show prolonged absolute but not relative healthspan}

318 Our data revealed that longevity mutants stay healthier compared to wild type and experience 319 a slower decline in physiological integrity. Indeed, dividing the lifespan of each genotype into three chronological fixed age categories: young (less than 7 days), middle (older than 8 but younger than 19 days), and old age ( $>20$ days of adulthood), showed a progressing decline of volume-corrected work performed (Fig. 6a, 6b). Longevity mutants performed better in the middle age group than wild type, but only daf-2(e1368) outperformed wild type in the old age 
324 group (Fig. 6a, 6b). Using hierarchical clustering of temporally scaled phenotypes as a complementary analysis, we found that longevity mutants often cluster with chronologically younger wild-type samples (Extended Data Fig. 7).

Integration of voluntary movement and forced maximum muscle strength quantification

Having two independent assessments of healthspan that act on very different stringency levels,

Consistent with previous observations in other species, the maximum power drops prior to the

338 longevity genotypes, daf-2(e1368) appears to be the healthiest strain (Fig. 6c). Thus, combining

339 multiple matrices of physiological and behavioral integrity is a powerful assessment of 340 healthspan. 


\section{Discussion}

344 Understanding the relationship between healthspan and lifespan is an important question in 345 aging research since geroscience aims to increase the time spent in good health and to postpone and compress the time suffering from age-related pathologies and chronic diseases $2,4,5,37$.

347 Model organisms like $C$. elegans are used to identify longevity-promoting interventions that 348 can then be of translational value for humans ${ }^{13,15}$. There is a fierce debate whether C. elegans 349 longevity interventions show compression of sickspan and are of translational value for 350 improving healthspan or healthy aging in humans ${ }^{16-20}$. In this study, we set out to develop a 351 robust method to quantify maximum muscle strength as a highly interpretable healthspan metric 352 with translational value. Lifespan measurements are well-established and allowed the study of 353 hundreds of lifespan-extending compounds and genetic alterations, leading to ground-breaking 354 discoveries. However, this development is not reflected in the area of healthspan extension. 355 Numerous methods exist which often measure proxy phenotypes for healthspan that are also motivation dependent like pharyngeal pumping, thrashing, and others. With our approach, we were able to directly quantify muscle health in $C$. elegans. This is especially relevant since $C$. elegans is an ideal model system for large-scale genetic screening and both the microfluidic device as well as the image detection can be multiplexed. This approach could translate to the much-needed identification of (muscle) health-promoting interventions.

The microfluidic device operates using acoustophoresis to generate an acoustic force field to quantify the physical fitness and muscle strength of aging $C$. elegans. Using six different ways to assess healthspan in forms of voluntary movements, swimming endurance, stress resilience, muscular force, muscular fatigue, structural integrity/compressibility, and quantifying several age-related morphological changes, including cuticle/skin wrinkles, body, and internal organ sizes, we find that most of these phenotypic changes are postponed in longevity mutants. We observed a hierarchical and time-dependent succession in the occurrence of these phenotypes, 
starting with a loss of stress resilience, then a loss of swimming endurance, decline in maximal

force as indicated by overcoming acoustic field (omega turns), decline in dynamic power,

371 seizing of body and organ growth (intestine), and then decline in voluntary movement and

372 becoming inactive and lethargic. Using principle component analysis, we show that many of

373 these phenotypes are strongly cross-correlated. The delay of all these age-related phenotypic

374 changes is evident when using chronological age as a reference point for comparison of

375 longevity mutants with wild type but disappears when using relative age as a reference. This

376 points to the idea of temporal scaling of the healthspan. Consistent with this idea, we find that

377 sickspan is neither compressed nor prolonged in longevity mutants compared to wild type.

378 Thus, our quantifications suggest that C. elegans healthspan undergoes temporal scaling in

379 longevity.

Aging is defined as a set of phenotypes or senescent pathologies occurring with a higher proportion in older individuals ${ }^{38}$. Which senescent pathologies limit the lifespan depends on our chosen set of phenotypes assessed are directly limiting or affecting lifespan is unclear.

However, it is evident that not one single mechanism underlies all our measured age-related phenotypes. On the other extreme, we do not observe a "one mechanism causing one agerelated pathology" mechanism. Our data shows that some phenotypes correlate and also follow interconnected. This favors a mixed model of several causal mechanisms affecting multiple connected and independent senescent pathologies/phenotypes, including lifespan limiting phenotypes ${ }^{38,39}$. Even if we might not measure lifespan limiting phenotypes directly, the related pathologies.

The lifespan of $C$. elegans can be increased by up to ten-fold ${ }^{40}$ and decreased by 40 394 fold, but surprisingly the lifespan curves often follow the same rescaled distribution ${ }^{41}$. 
Temporal scaling was also noted when comparing expression profiles of longevity mutants compared to wild-type C. elegans ${ }^{42}$. Aging is thought to be caused by the accumulation of

397 damage 14,43 . Organisms either remove, detoxify, compartmentalize, or dilute molecular 398 damage ${ }^{43}$. In dividing stem cells, yeast, or bacteria, damaged macromolecules or organelles 399 are kept in the "mother" cell leading to accumulation of damage ${ }^{14,44,45}$. Since C. elegans 400 somatic cells are post-mitotic, the main driver of aging dictating the lifespan of C. elegans might be the accumulation of damage in non-dividing cells. In a perfect life-conditioned environment such as the laboratory setting, free from ever-changing environmental fluctuations or pathogens, the only lifespan limiting factor probably boils down to the imperfectness of biological systems ${ }^{43}$, leading to accumulation of damage, stochastic decay of homeostatic mechanisms ${ }^{14}$, and consequently to age-related pathologies and phenotypes. In light of this, it might be less surprising that the onset of pathologies is strongly linked to lifespan and thereby, healthspan is rather temporally scaled than prolonged in longevity. in the future. However, certain longitudinal C. elegans phenotypes are comparable to human age-related phenotypes. Analogous to the $C$. elegans volume increase to peak mid-age and then decrease is that human BMI and waist circumference also follows this early-to-mid-life increase reaching a peak around 65-70 years and then declining ${ }^{46}$. Furthermore, grip strength progressively declines after the age of $30-40^{46}$, similarly to C. elegans muscular strength. This

414 raises the question of whether non-compression of sickspan observed in C. elegans means or 415 interpolates to non-compression of sickspan in humans? Since aging is universal, it is tempting 416 to speculate that the underlying mechanisms of aging or age-dependent phenotypes are also 417 universal. This might be a potentially erroneous or unproven extension of the observation that 418 almost all living things age ${ }^{38}$. Although phenotypic changes, such as the loss of C. elegans 
underlying biological mechanisms resulting in physical weakness might be different. Our study

421

422

423

424

425

426

427

428

429

430

431

432

433

434

435

436

437

makes no conclusion or interpolation about the compression of the sickspan in humans.

There is an accumulating body of evidence that long-lived humans are healthy during old age. For instance, $56-83 \%$ and $15-23 \%$ of centenarians, people over the age of hundred years, delay the onset of chronic age-dependent diseases and physical disabilities or were even free of such co-morbidities and frailties, respectively ${ }^{7,10}$. Centenarians have lower incidence rates of chronic illnesses compared to their 90 - or 80 -year old matched-controls ${ }^{8,9,11,12}$. This also extends to family members related to centenarians compared to families without centenarians ${ }^{47,48}$. Thus, centenarians have a later onset and a lower rate of incidence compared to people in their eighties, similar to our observation when comparing longevity mutants to wild-type $C$. elegans. However, since centenarians get to enjoy at least 20 more years, how would this comparison look if we were to compare relative age to chronological age? Is sickspan compressed or temporally scaled in centenarians compared to the average population? As our life expectancy doubled in the last hundred years and we are on the course of potentially reaching the limit of our lifespan ${ }^{37}$, the accompanied delayed onset of disabilities already started to decelerate in longer-lived woman compared to men ${ }^{49}$. On the other hand, there are several interventions that increase healthspan without increasing lifespan per se identified in mice ${ }^{50,51}$ and Rhesus monkeys ${ }^{52}$. Thus, studying longevity is an important first step of identifying molecular mechanisms promoting healthy aging, but our study and others ${ }^{50-52}$ point toward that it is crucially important for geroscience to start investigating interventions that improve healthspan directly in future studies ${ }^{6}$. Initial steps in defining healthspan ${ }^{4,5}$ and also tools and experimental setups, including this study, are being developed to reliably quantify healthspan ${ }^{53-57}$.

In summary, we have demonstrated that $C$. elegans sickspan is neither compressed nor extended in longevity mutants providing an alternative answer to an ongoing debate in the aging field. With our measurements, we showed that previous claims that insulin/IGF-1 receptor mutants 
446 have increased sickspan compared to wild type are correct if the voluntary movement is

447 measured, but not the case if the muscular function or other healthspan measurements are

448 considered that do not rely on the behavioral state of the animal. By adjusting the reference

449 system from chronological age to relative age, we provide evidence that the healthspan of

450 longevity mutants undergoes temporal scaling. Future studies using our acoustophoresis

451 approach to study the role of healthspan will reveal novel strategies to improve healthy aging.

452

453 


\section{Author contributions}

455 All authors participated in analyzing and interpreting the data and designing the experiments.

456 CS performed lifespan assays. CYE and CS performed swimming and oxidative stress assays.

457 PR designed and performed microfluidic assays. CS and PR analyzed the datasets jointly. CS

458 and CYE wrote the manuscript in consultation with the other authors.

459

460 Author Information

461 The authors have no competing interests to declare. Correspondence should be addressed to C.

462 Y. E. and J.D.

463

464 Acknowledgment

465 We thank the Ewald lab for constructive comments on the manuscript. Some strains were

466 provided by the CGC, which is funded by NIH Office of Research Infrastructure Programs (P40

467 OD010440). Funding from the Swiss National Science Foundation PP00P3_163898 to CYE 468 and CS.

469 
bioRxiv preprint doi: https://doi.org/10.1101/2021.05.31.446397; this version posted May 31, 2021. The copyright holder for this preprint (which was not certified by peer review) is the author/funder, who has granted bioRxiv a license to display the preprint in perpetuity. It is made available under aCC-BY-NC-ND 4.0 International license.

A

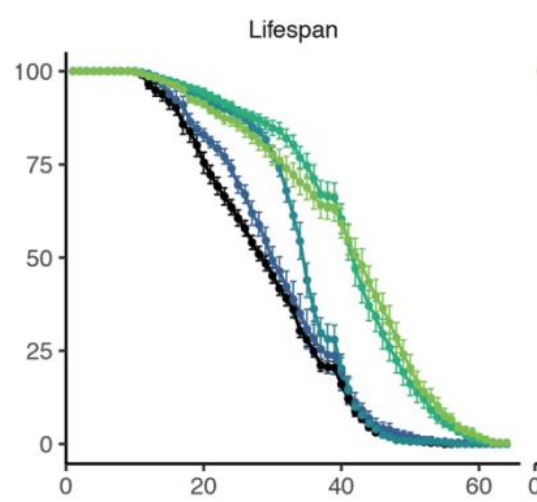

B

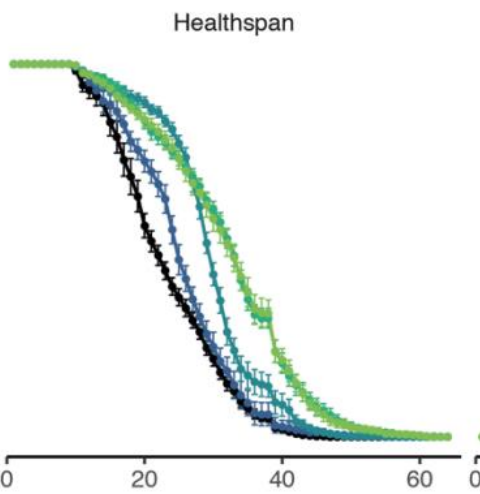

C

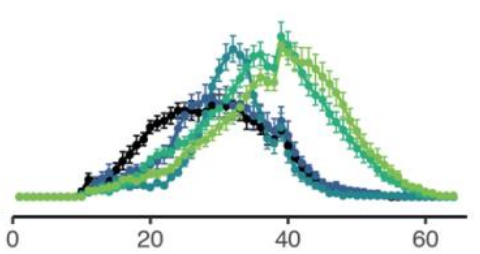

D
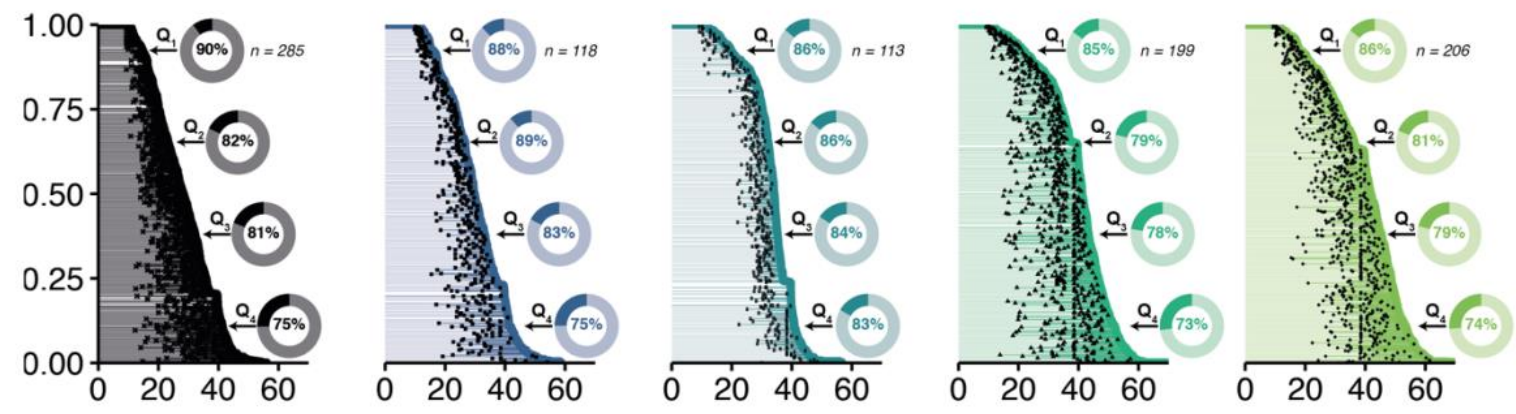

$\mathrm{E}$

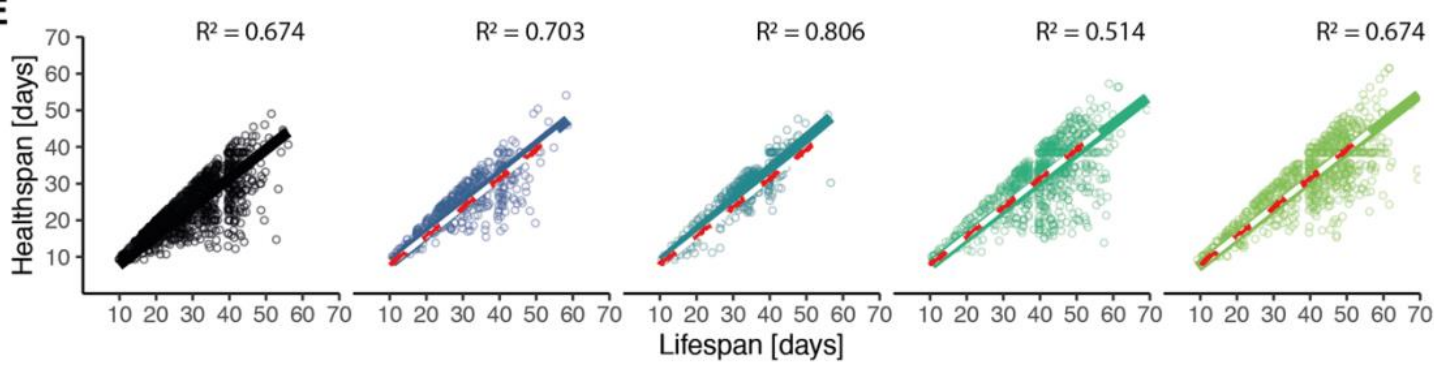

$\mathbf{F}$

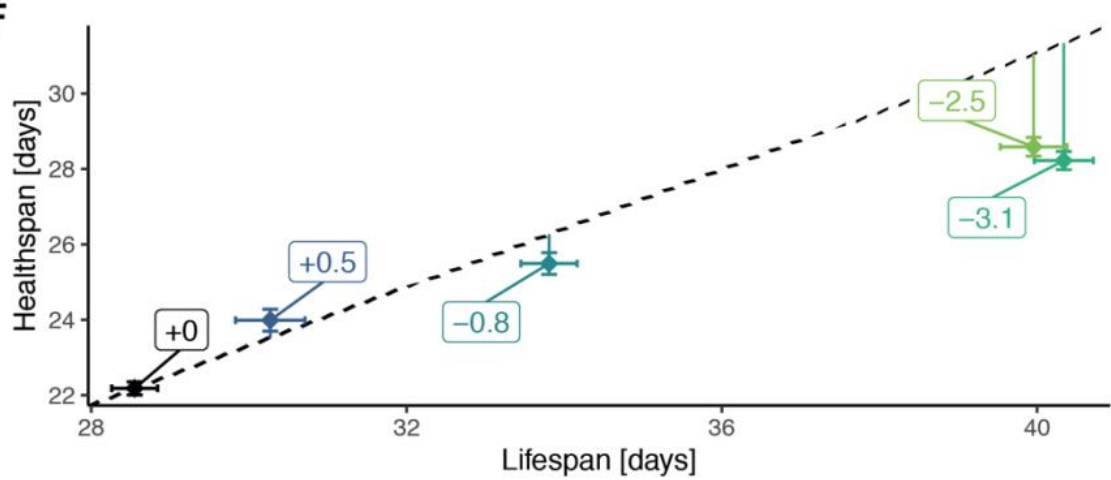

Genotypes

N2 (wild type)

eat-2

$g / p-1$

daf-2(e1368)

daf-2(e1370)

Lifespan [days]

Figure 1: Voluntary movement quantification in aging C. elegans populations. C. elegans lifespan analysis displaying survival (A), healthspan $(B)$, and sickspan $(C)$ for each genotype. Error bars display the standard error between plates at $24 \mathrm{~h}$ intervals. Healthspan refers to the timespan of fast movement, sickspan to the time spend in sedentary movement, and lifespan to the time until the animal fails to move irretrievably. The fate of each individual is displayed separately for each genotype overlayed with the population's survival $(D)$. Here, each individual's healthspan is marked as a transparent line spanning from young adulthood to the onset of sickspan marked by a black dot and then extends further as sickspan until the individual's point of death on the population survival curve. The inset displays the overall proportion each genotype spends in their healthspan for each lifespan quantile $\left(Q_{1}-Q_{4}\right)$. The correlation between health and lifespan is shown in figure $(E)$. Each individual is represented as a point with its lifespan on the $x$-axis and its corresponding healthspan on the $y$-axis. A linear model passing through the origin is shown as a solid line. The N2 model is superimposed on the longevity mutants as a red and white dashed line. All genotypes are compared to temporarily scaled N2 with the mean population life-and healthspan and error bars indicating the standard errors $(F)$. The extrapolated ratio of health- to the lifespan of wild type 
bioRxiv preprint doi: https://doi.org/10.1101/2021.05.31.446397; this version posted May 31,2021 . The copyright holder for this preprint

(which was not certified by peer review) is the author/funder, who has granted bioRxiv a license to display the preprint in perpetuity. It is made available under aCC-BY-NC-ND 4.0 International license.

A
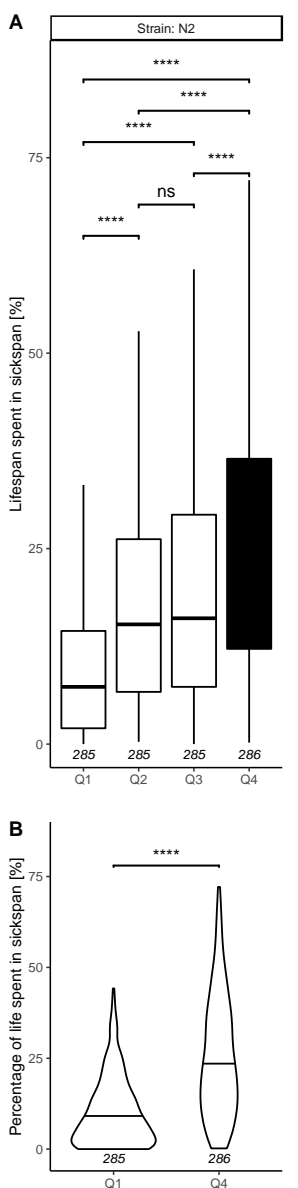
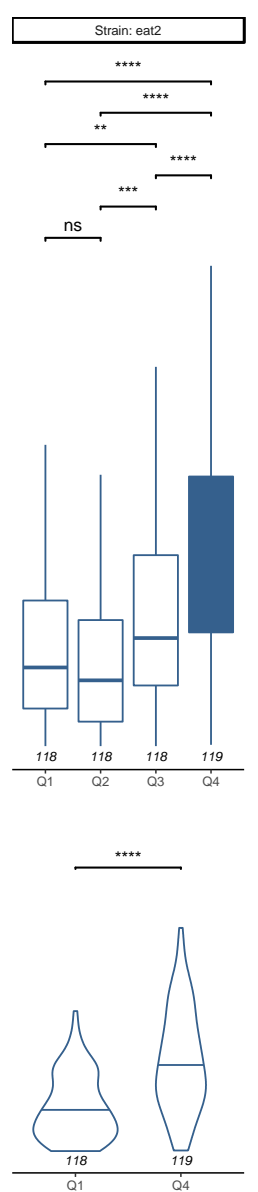
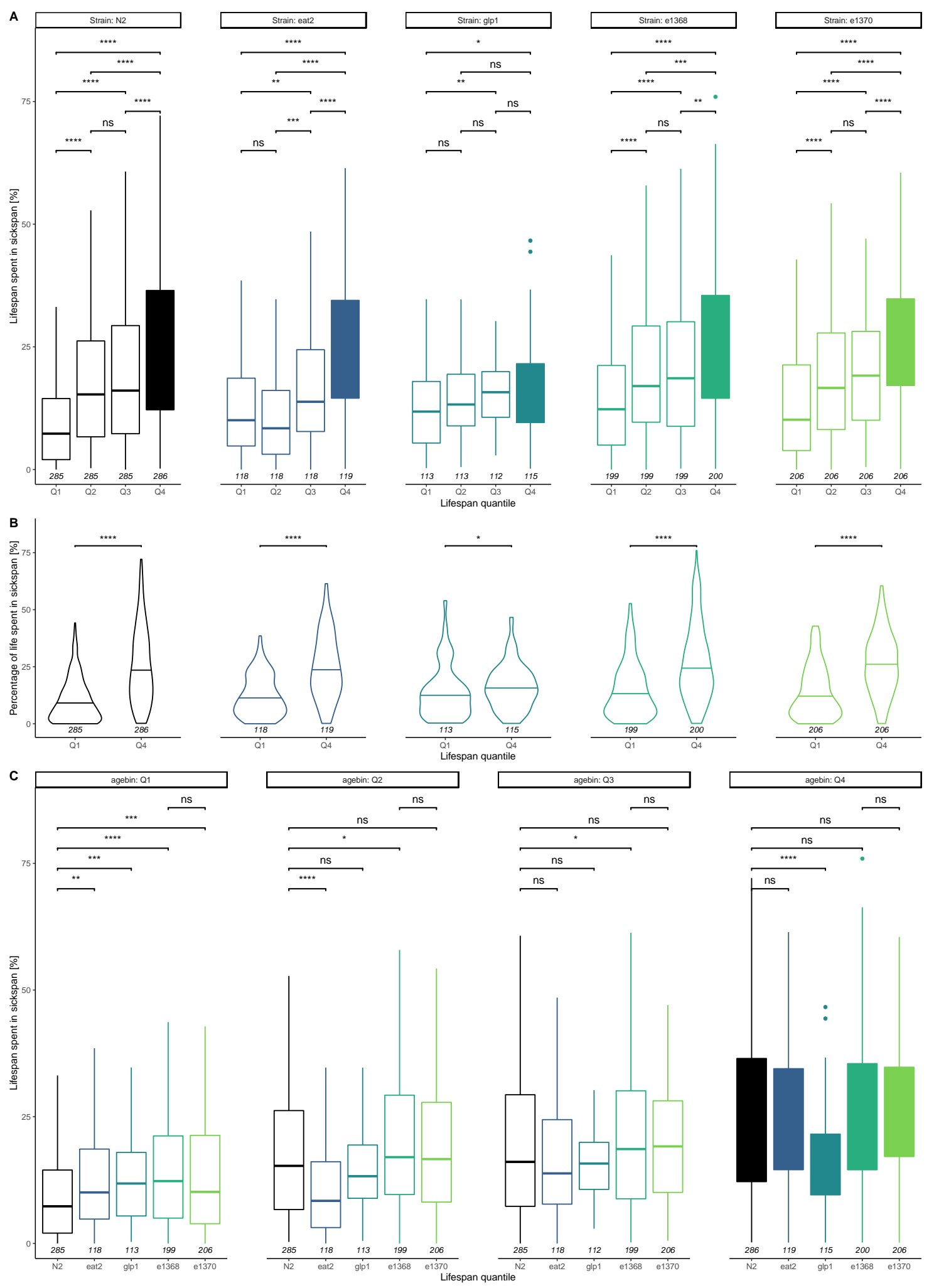

Strain

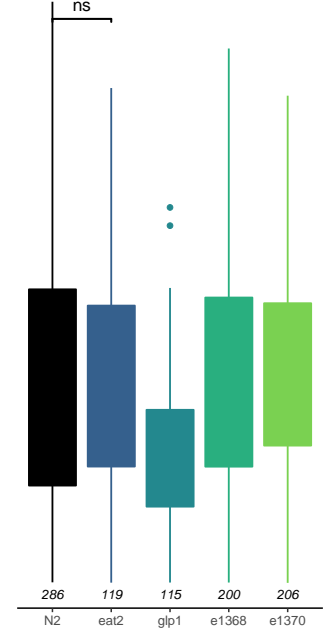

Extended Data Fig. 1: Paradoxon - young animals die apparently in good health.

The sickspan distribution for every quartile of the lifespan distribution is displayed as boxplots with each strain in a separate 
bioRxiv preprint doi: https://doi.org/10.1101/2021.05.31.446397; this version posted May 31, 2021. The copyright holder for this preprint (which was not certified by peer review) is the author/funder, who has granted bioRxiv a license to display the preprint in perpetuity. It is made available under aCC-BY-NC-ND 4.0 International license.

panel (A). Animals experience progressively higher sickspan ratios with each quantile, with the two middle quantiles being the most similar. The distributions of the first and fourth quartile are shown as violin plots with their median line highlighted by a horizontal segment showcasing the increase of relative sickspan and heterogeneity with age across genotypes (B). To compare the individual genotypes for each relative age cohort, the corresponding quartiles are contrasted as boxplots for each strain and each quartile in a separate panel and indicate temporal scaling of healthspan $(C)$. The sickspan ratio is compared across genotypes and quartiles (Mann-Whitney test) and the P-values are depicted as symbols ( $n s>0.05, *$ $0.05, * *<0.01, * * *<0.001, * * * *<0.0001)$. When all quartiles are displayed, the median sickspan percentage of the first and last quartile of the wild type population are indicated as dashed horizontal lines. The number of observations associated with every subpopulation is displayed at the bottom of each observation. Each strain is shown in its distinct color, and increasing age is reflected by increasingly dark coloring.

500 
bioRxiv preprint doi: https://doi.org/10.1101/2021.05.31.446397; this version posted May 31, 2021. The copyright holder for this preprint (which was not certified by peer review) is the author/funder, who has granted bioRxiv a license to display the preprint in perpetuity. It is made available under aCC-BY-NC-ND 4.0 International license.

A

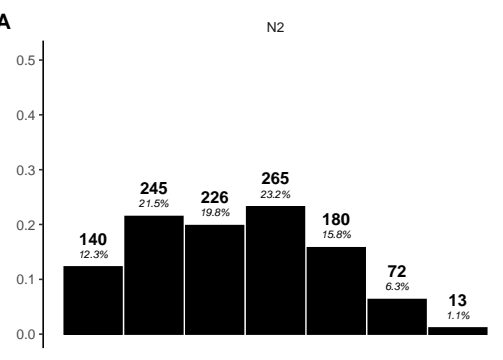

eat2

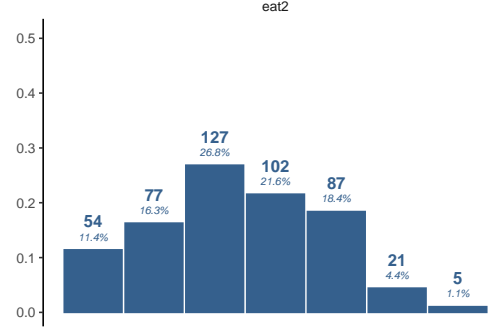

glp1
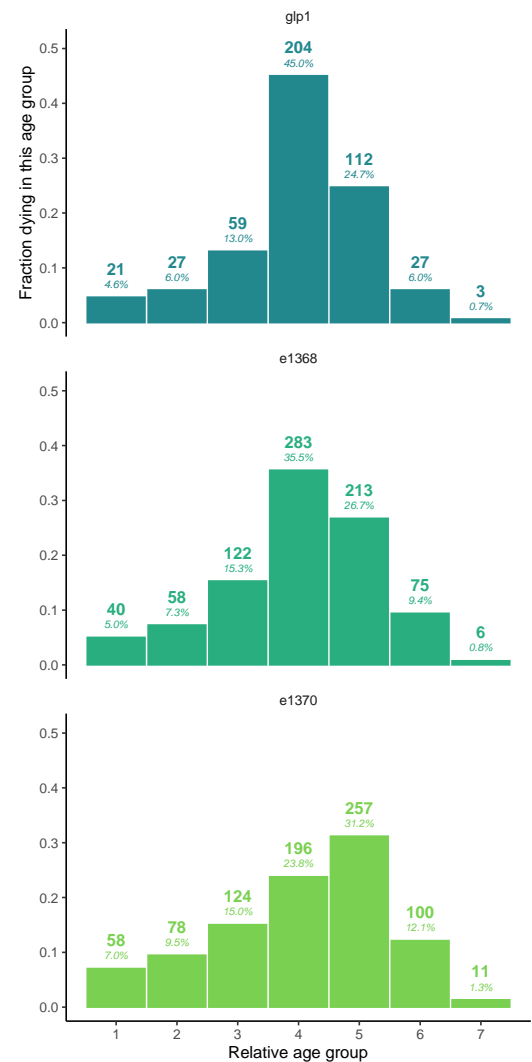

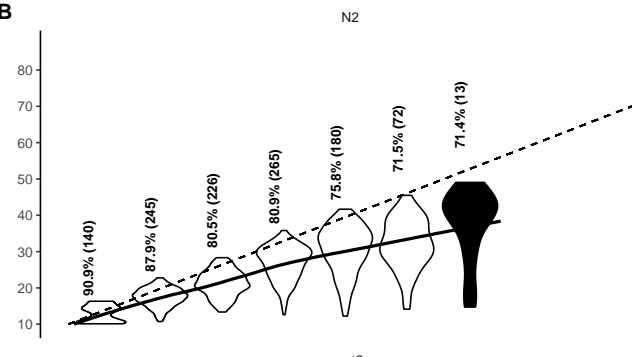

eat2

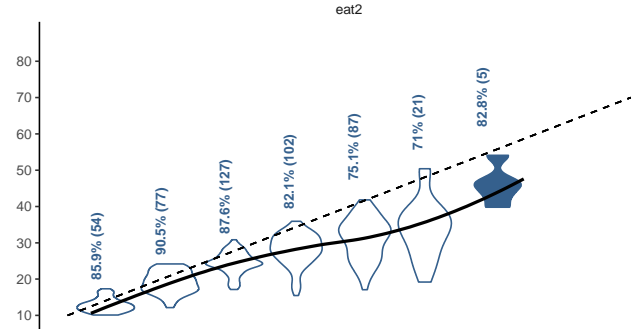

glp1

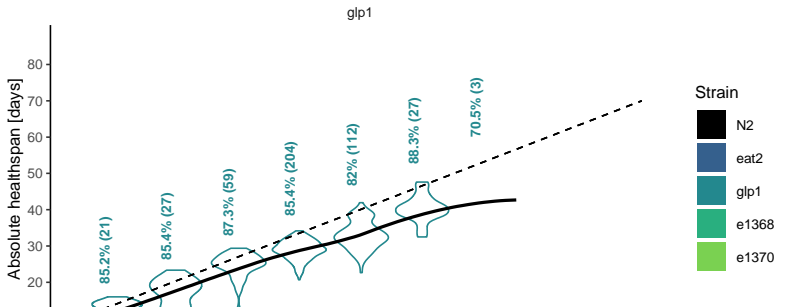

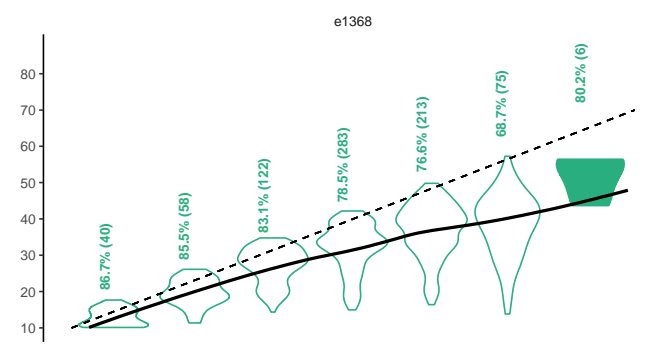

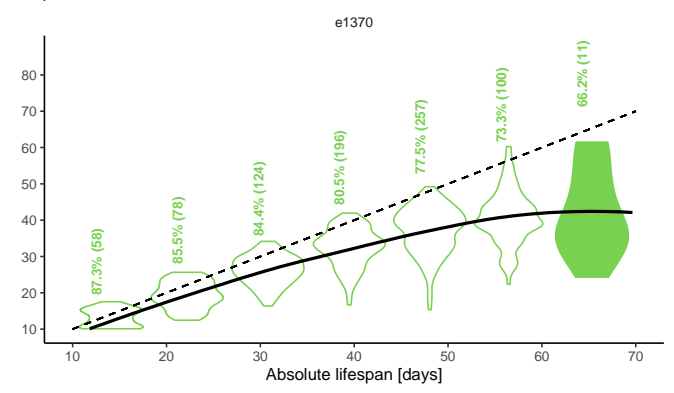

Extended Data Fig. 2: Relative healthspan within early and late dying C. elegans groups.

To address the question whether outliers drive the overall health-to-lifespan ratio, each genotype was divided into seven groups with equally sized age bins between the first and last recorded death in each genotype's population to visualize the distribution of death events. At the top of each bar, the number of animals observed to die in this cohort and its share in the overall population is displayed $(\boldsymbol{A})$. The healthspan distribution in each age group is shown as a violin plot $(\boldsymbol{B})$. The average percentage of each population spent in their healthspan is displayed above each group, with the population size indicated in brackets. The health-to-lifespan diagonal is shown as a dashed line, and a loess trendline was fitted to the binned datasets with a $95 \%$ confidence interval shaded in grey. In case the sample size falls below a threshold, the violin plot was omitted. Each analysis is displayed separately for every C. elegans genotype, which are each shown in a separate line and in a distinct color. The spread of the healthspan distribution increases at older ages, with some animals experiencing nearly no sickspan and others spending most of their life in the sickspan portion. 
bioRxiv preprint doi: https://doi.org/10.1101/2021.05.31.446397; this version posted May 31, 2021. The copyright holder for this preprint (which was not certified by peer review) is the author/funder, who has granted bioRxiv a license to display the preprint in perpetuity. It is made available under aCC-BY-NC-ND 4.0 International license.

a)

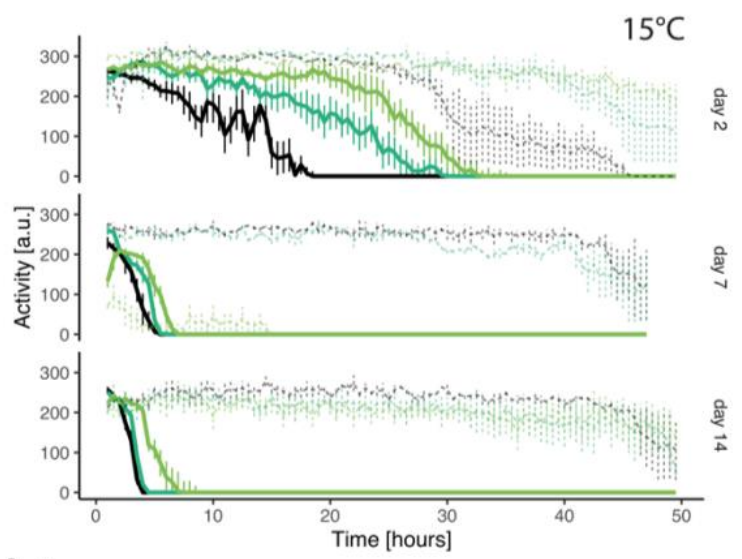

\section{Genotypes \\ N2 (wild type) \\ daf-2(e1368)}

daf-2(e1370)

\section{Genotypes}

spe-9 (control)

gip-1(e2141)

spe-9; daf-2(e1370)

b)
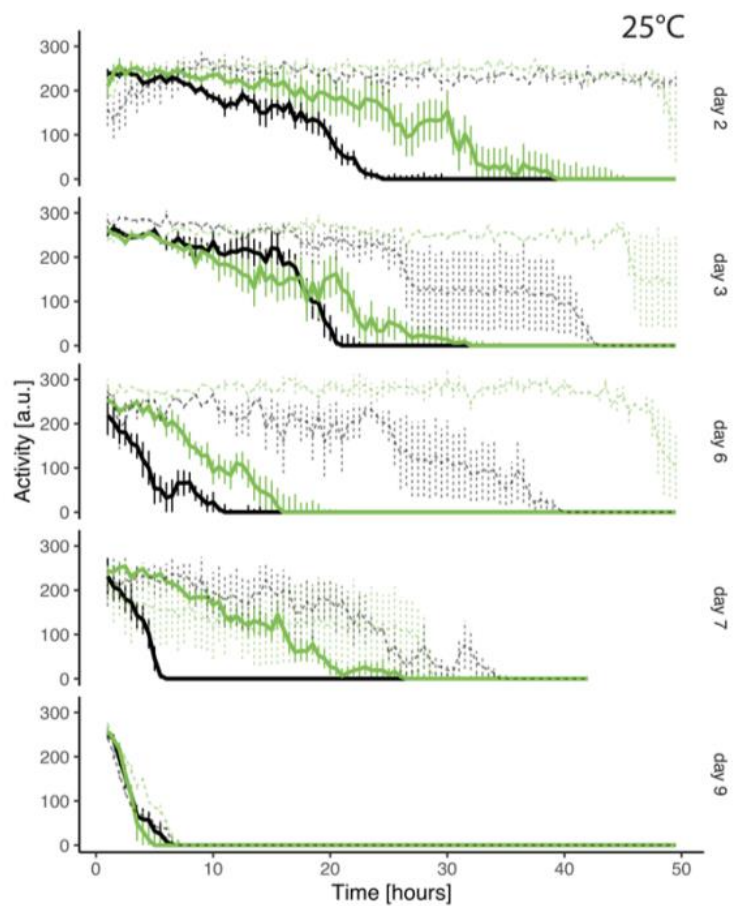

c)

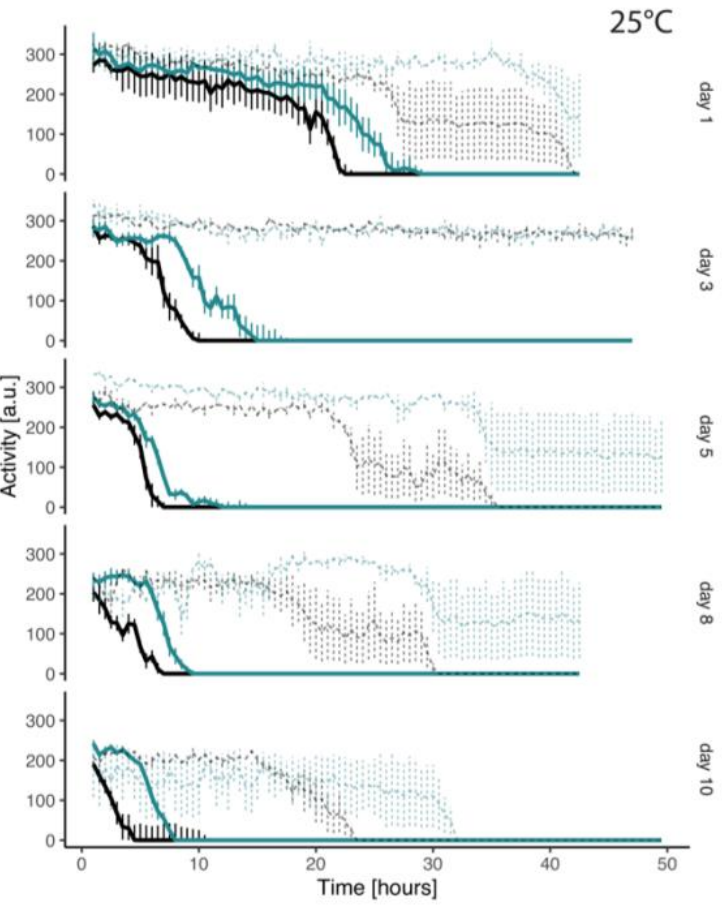

Figure 2: Progressive movement decline with age in unchallenged swimming assay and when exposed to arsenite.

In all panels, the time after subjecting the C. elegans populations to the arsenite stress is displayed on the $x$-axis and the movement activity of animals on the $y$-axis. The animal age at the start of the experiment is shown at the right of every panel. The mean activity in $14 \mathrm{mM}$ arsenite is shown as a continuous line and in $M 9$ as dashed lines. M9 was selected to avoid issues related to osmolarity. Vertical lines reflect the standard deviation at every measurement point.

Two daf-2 alleles and $\mathrm{N} 2$ populations were maintained at $15^{\circ} \mathrm{C}$ and subjected to movement quantification at three aging time points (a). The daf-2(e1370) allele was also assayed in a spe-9 background and therefore maintained at $15^{\circ} \mathrm{C}$ until L3 and then shifted to $25^{\circ} \mathrm{C}$ until the start of the assay $(b)$. The glp-1(e2141) strain was compared against a spe-9 population and maintained at $25^{\circ} \mathrm{C}$ after population lysis until the time of measurement. 
bioRxiv preprint doi: https://doi.org/10.1101/2021.05.31.446397; this version posted May 31, 2021. The copyright holder for this preprint (which was not certified by peer review) is the author/funder, who has granted bioRxiv a license to display the preprint in perpetuity. It is made available under aCC-BY-NC-ND 4.0 International license.

A

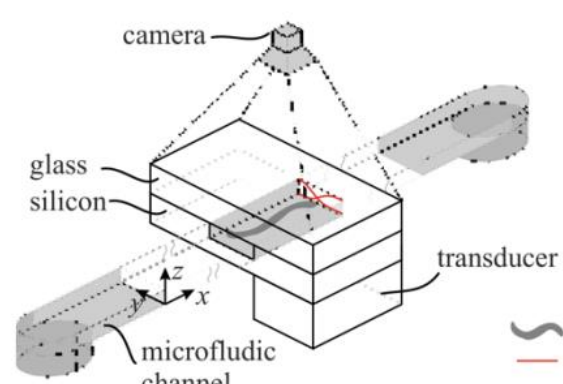

C. elegans standing wave
B
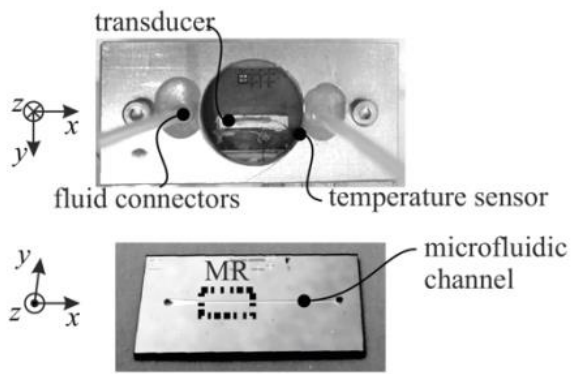

(3) dorsal muscle contraction

(4) turnaround
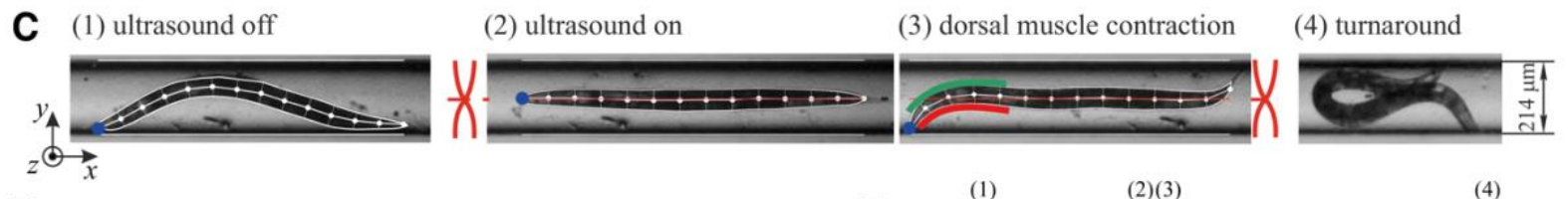

D

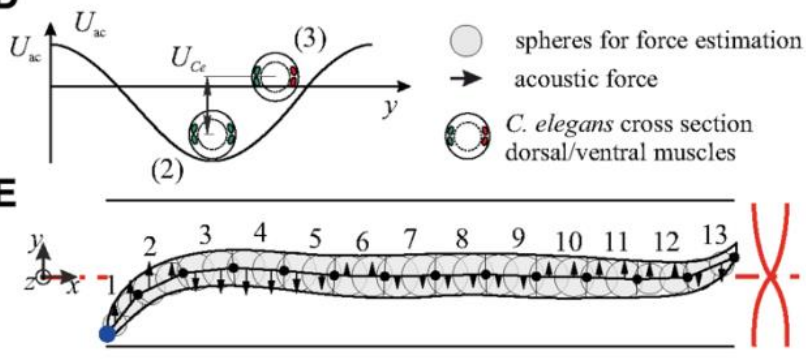

G

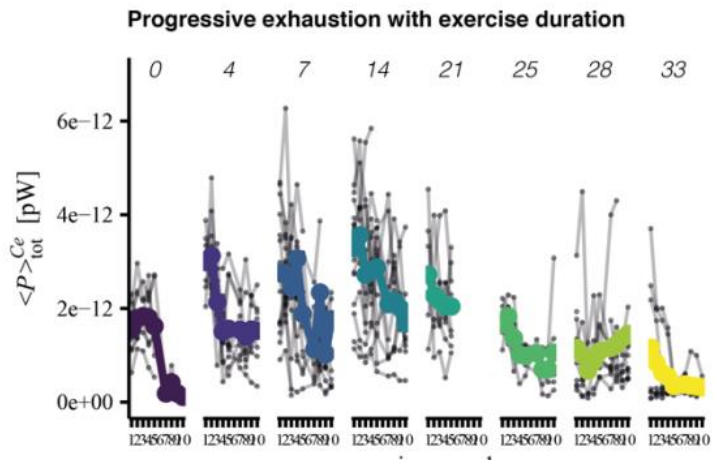

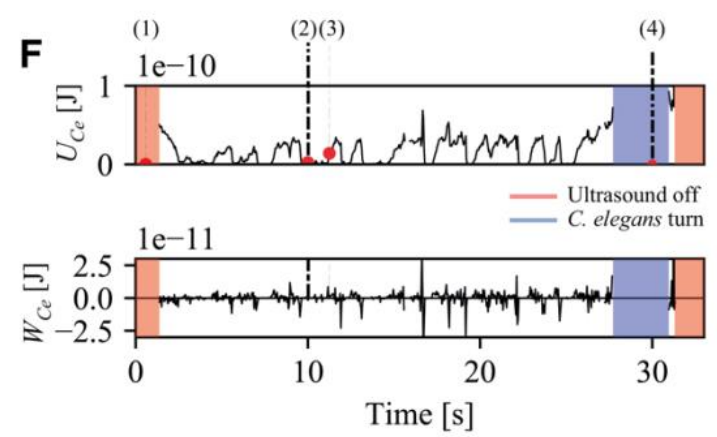

H Progressive exhaustion with exercise duration

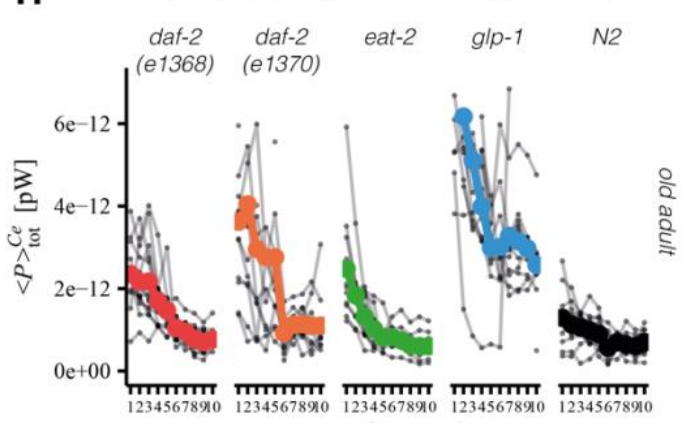

exercise round

Figure 3: Experimental setup to measure maximum C. elegans muscle strength:

Top view of the schematic representation of the silicon/glass acoustofluidic chip, fluid in- and outlets, and the piezoelectric transducer positioned at the bottom and C. elegans trapped in the standing wave (A). A photograph of the back of the chip together with the metal clamps, the attached fluid connectors, temperature probe, and a piezoelectric transducer is shown (B, top). The front view of the chip provides an overview of the device shape, the microfluidic channel, and the measurement region $(M R)(B$, bottom). A day 4 wild type $C$. elegans, as seen by the camera in the measurement region, is displayed together with the image processing output highlighting the outline and the segmented midline of the animal as well as the channel borders and centerline $(C)$. The effect of the acoustic field (frequency: $3.543 \mathrm{MHz}$, voltage amplitude: $76 \mathrm{Vpp}, \lambda / 2$ mode) is shown in $(C, 2)$, aligning the animal at the midline. The animal exercises maximum muscle power attempting to bend its head away from the midline $(C, 3)$ to achieve a turn $(C, 4)$, likely as an attempted escape response. The working model to quantify the C. elegans muscular force consists of 13 rigid links along the animal's midline, which are connected by joints. Blue arrows reflect the muscle activity acting on each joint to generate a force against the acoustic field $(E)$. The acoustic force acting along the animal's body is modeled as the individual acoustic forces acting on the grey spheres aligned along the body and represented by black arrows, with the length of each arrow being proportional to its force or moment magnitude. The acoustic radiation potential is illustrated using a C. elegans cross-section highlighting the animal's four body wall muscles (D). If stretched, the animal rests at the minimum (see C 2) and, upon muscle contraction, moves upwards in its potential energy well (see C3). The four C. elegans frames (C 1-4) are put in the context of one 30 seconds actuation cycle showing the time-resolved total energy and mechanical work quantification for this animal $(F)$. Red areas reflect regions of zero energy due to the acoustic field being turned off, and the blue areas indicate C. elegans turn movements during which muscle force estimation is paused. A C. elegans maximum force assessment routine consists of multiple 30 seconds exercise rounds intermitted by 5-second breaks for a total of up to ten actuation cycles. For wild type, this exercise regimen is displayed with the exercise rounds on the $x$-axis, the total power (time-averaged) on the y axis, as derived from the respective total energy curve in $(F)$, and the age of the population given at the top of each facet $(G)$. Wild type $C$. elegans is contrasted to long-lived C. elegans genotypes across up to ten exercise rounds focusing on the aged cohort above day 20 of adulthood $(H)$. 
bioRxiv preprint doi: https://doi.org/10.1101/2021.05.31.446397; this version posted May 31, 2021. The copyright holder for this preprint (which was not certified by peer review) is the author/funder, who has granted bioRxiv a license to display the preprint in perpetuity. It is made available under aCC-BY-NC-ND 4.0 International license.

N2 vs. daf-2(e1368)

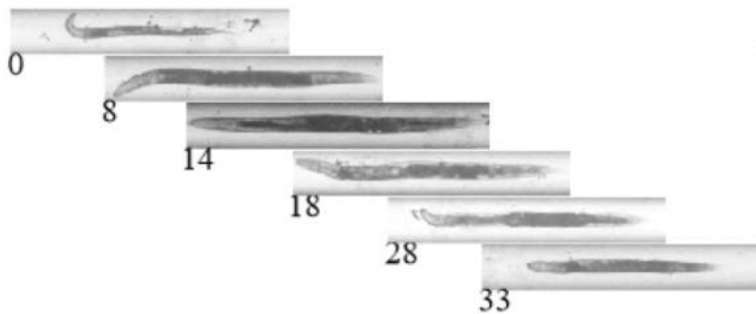

age [days]
A Median length of each individual animal

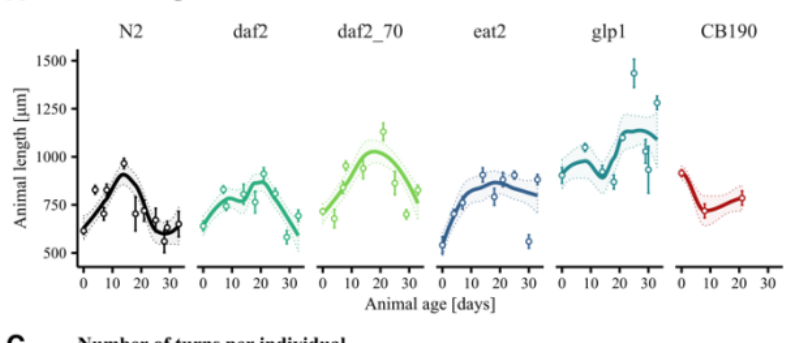

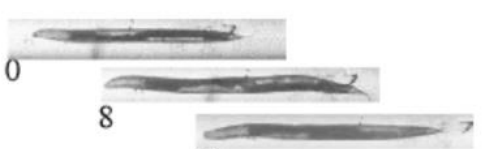

14
29
$33 \quad 400 \mu \mathrm{m}$
C Number of turns per individual

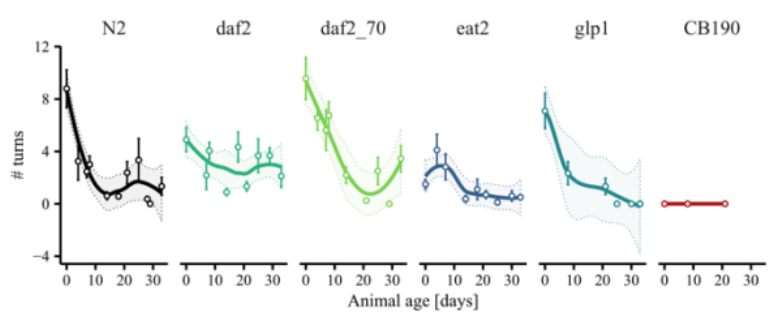

E Effect of the acoustic field on animal diameter

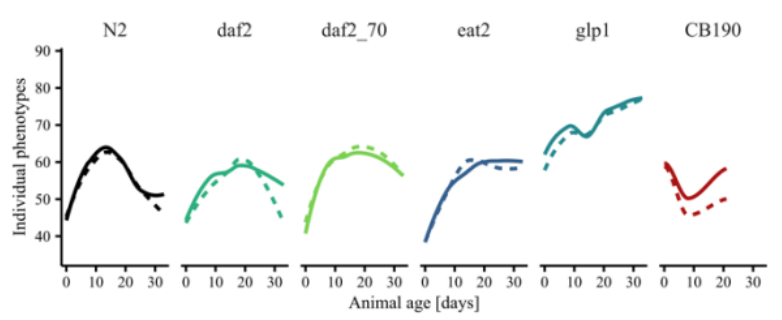

G Dynamic power/volume

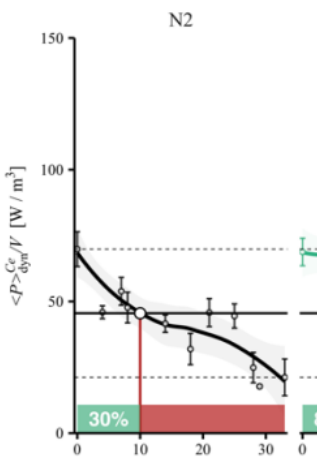

daf2 70

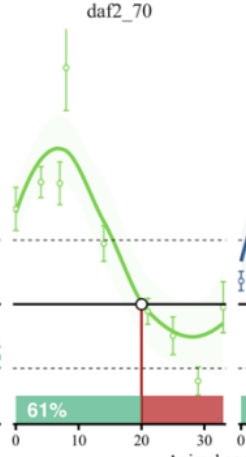

B Volume for each individual age [days]

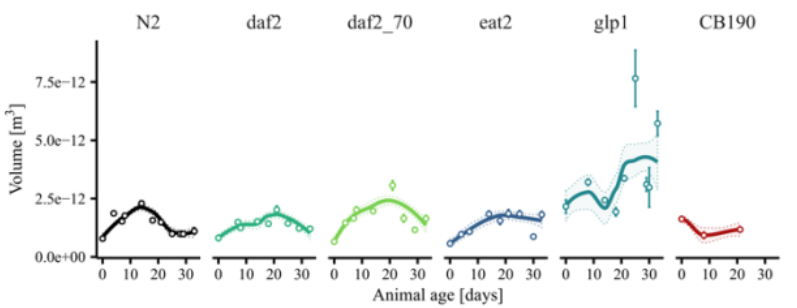

D Median energy per individual

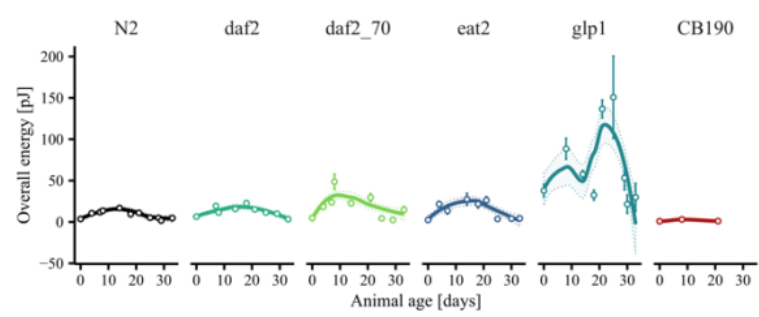

$\mathbf{F}$

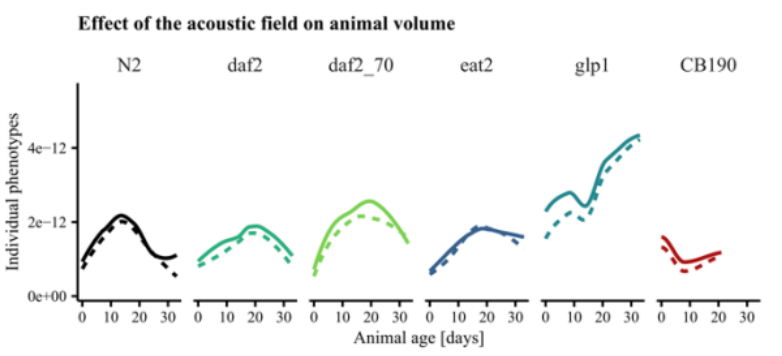

eat2

glp1

CB190
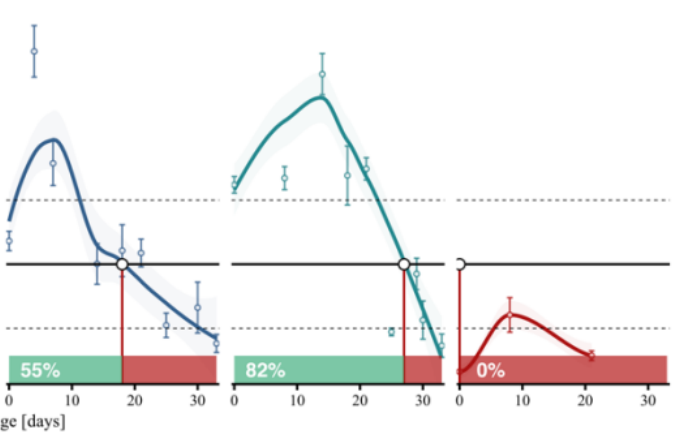

strain

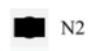

Figure 4: Time course of fitness- and structural-related phenotypes for wild type and long-lived C. elegans under acoustic stimulation.

Acoustic compression was used to quantify phenotypes directly (\# turns, energy, diameter-and volume compression, and dynamic power by volume) as well as indirectly by exploiting the non-destructive linear alignment of the animals (length, volume). Randomly selected images for $N 2(A)$ and daf-2(e1368) (B) at different ages are displayed to illustrate the positioning of the C. elegans in the microfluidic measurement channel and how all matrices are shown below were obtained. Measurements are displayed as mean \pm standard error for each assessed age point and subjected to local polynomial regression fitting displayed as a full line with the confidence interval set to $95 \%$ and bounded by dashed lines. For the acoustic compression only, the fitted line is shown. The length $(C)$ and volume $(D)$ of the animal was quantified automatically 
bioRxiv preprint doi: https://doi.org/10.1101/2021.05.31.446397; this version posted May 31, 2021. The copyright holder for this preprint (which was not certified by peer review) is the author/funder, who has granted bioRxiv a license to display the preprint in perpetuity. It is made available under aCC-BY-NC-ND 4.0 International license.

564

565

566

567

568

569

570

571

572

573

574

575

576

577

578

579

580

using the entire length and area of the animal in the channel, respectively. The number of turns was quantified manually and corresponded to the number of times an animal successfully changed its orientation in the channel by $180^{\circ}$. The total energy of the individual was calculated using the magnitude of the lateral deflection of the animal from the channel middle $(F)$. The compression experienced by the animal diameter $(G)$ and volume $(H)$ when the field is activated was computed automatically and displayed as a full line when the field is on and a dashed line when the field is off. The dynamic power/volume (I) reflects the work the animal performs against the field to change its lateral position in the channel and is normalized by the overall volume of the animal to enable comparisons across genotypes making this the most informative health parameter. The mean value for day 0 and day $33 \mathrm{~N} 2$ animals are indicated in black dashed lines, and the half-activity value between these two extremes is shown as a full line which is also used to deduce the health-to sickspan transition for each genotype. Using this $N 2$ half-activity value, the individual strains reach their sickspan at approximately 10 days for N2, 28 days for daf-2(e1368), 20 days for daf-2(e1370), 18 days for eat-2, and 27 days for glp-1 while CB190 spends its entire lifespan in its sickspan fraction. Long-lived genotypes, in general, do not experience the same linear energy density decline as wild type since their total energy decrease is slowed down, as is their growth leading to a non-linear energy density trajectory. 
bioRxiv preprint doi: https://doi.org/10.1101/2021.05.31.446397; this version posted May 31, 2021. The copyright holder for this preprint (which was not certified by peer review) is the author/funder, who has granted bioRxiv a license to display the preprint in perpetuity. It is made available under aCC-BY-NC-ND 4.0 International license.

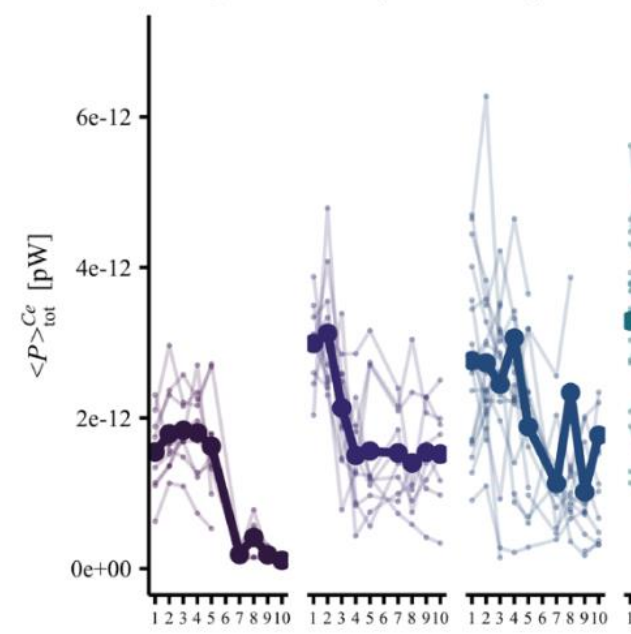

B Initial performance remains nearly constant
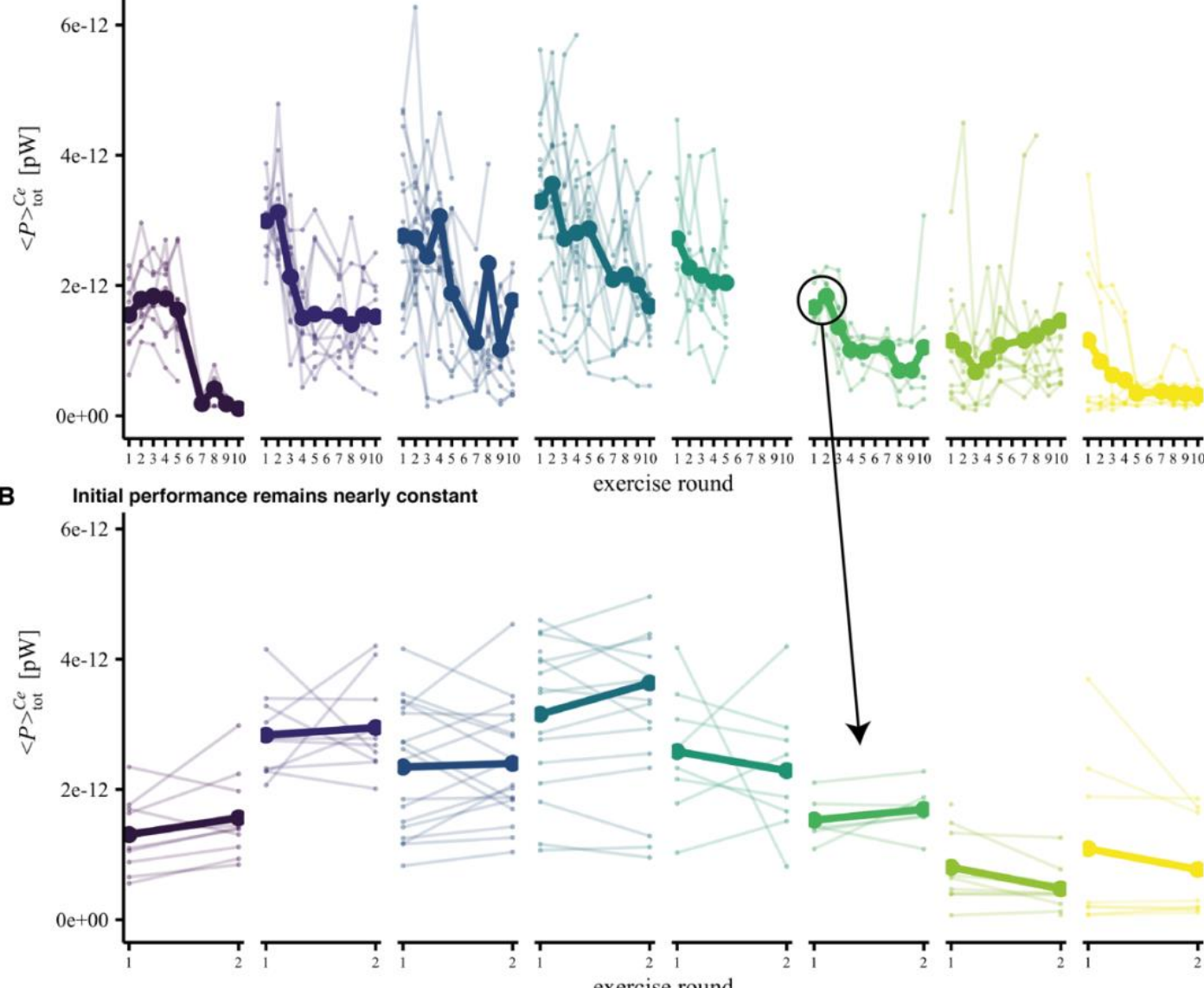

Age

C

Displacement across body spline Absolute displacement, randomly sampled N2, field on.

D Relative head displacement Day 29, population averages, shown as relative movement at the head

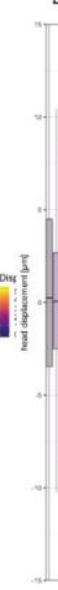

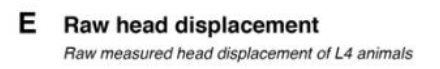

Raw measured head displacement of $L 4$ animals

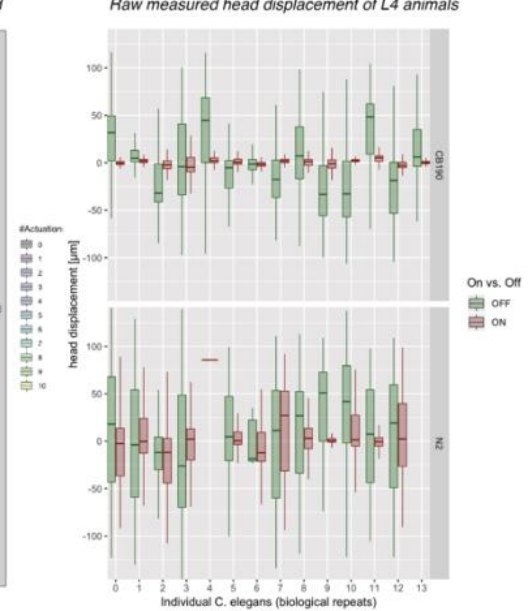

Extended Data Fig. 3. C. elegans movement in the acoustic exercise chamber.

The exercise regimen was benchmarked for wild type (N2) C. elegans at different ages to quantify after how many cycles they experience muscle fatigue $(A, B)$. Every trace corresponds to one individual animal that was up to ten times subjected to 30 seconds maximum force exercise followed by a 5 seconds break (A). Thicker lines represent the population means at every time point. To quantify maximum muscle strength, we selected the first two actuation cycles $(B)$ at which no fatigue can yet be identified. The total energy is computed over the entire body and muscle apparatus of the animal. Interestingly, the spline position most activated in this exercise is the head and tail sections $(C)$. Focusing on the head we quantified the relative head movement for different strains at old age and also observed the same trend of decreasing movement with higher actuation cycles (D). The extreme case of the paralyzed CB190 vs. N2 in young adulthood displays that while the mutant is still able to move the head away from the midline of the field when the acoustic field is off, it is unable to move when the field is on while $N 2$ successfully fights against the applied forces $(E)$. 
bioRxiv preprint doi: https://doi.org/10.1101/2021.05.31.446397; this version posted May 31, 2021. The copyright holder for this preprint (which was not certified by peer review) is the author/funder, who has granted bioRxiv a license to display the preprint in perpetuity. It is made available under aCC-BY-NC-ND 4.0 International license.

593

A

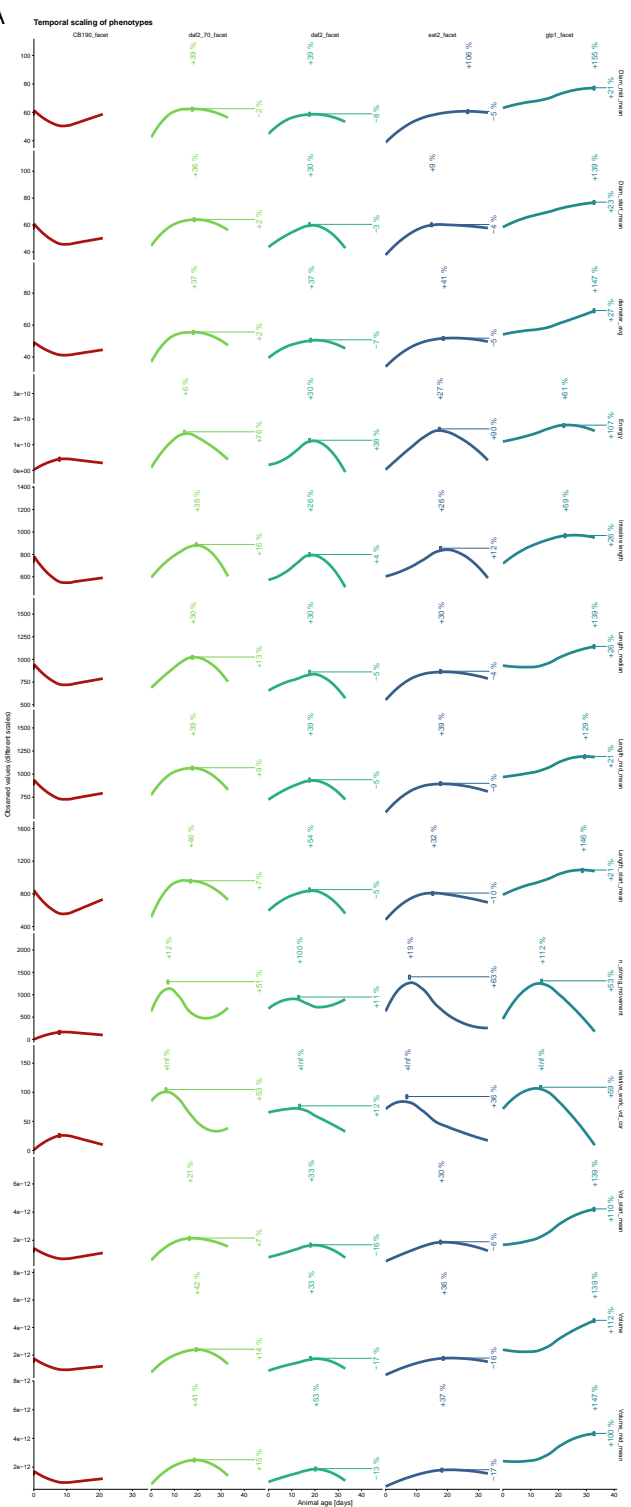

B
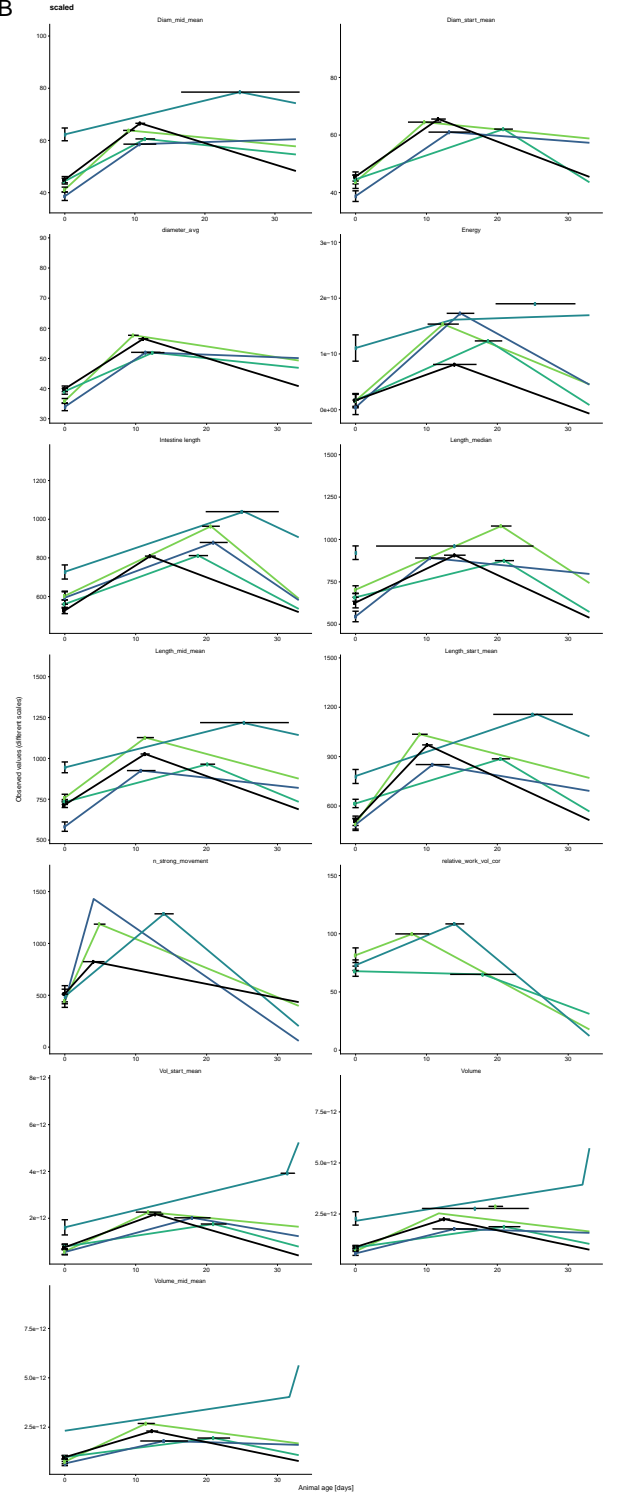

Figure 5.Temporal scaling of C. elegans aging phenotypes.

The measured values of selected phenotypes are shown using different scales on the y-axis, animal age on the $x$-axis, and facetted by strain (A). To address the temporal scaling of phenotypes hypothesis, the loess fit of the measured values for wild type is shown as a solid grey line, and it is temporally scaled values using the respective mean lifespan increase experienced by the respective strain are shown as a dashed line also in grey. The measured phenotype trajectory for each strain is shown in color, each in a separate panel. The maximum fitted value is marked using. White point and its increase relative to the maximum fitted values measured for wild type are shown for both the age and phenotype variables. The same phenotypes as in subfigure $(C)$ are modeled using a piecewise linear relationship in $(B)$. The breakpoint of the segmented fit is estimated by the model at the age value where the linear relationship between the measured phenotype and population age changes. The individual animals measured at each time point are displayed as mean +/- standard error. 
bioRxiv preprint doi: https://doi.org/10.1101/2021.05.31.446397; this version posted May 31, 2021. The copyright holder for this preprint (which was not certified by peer review) is the author/funder, who has granted bioRxiv a license to display the preprint in perpetuity. It is made available under aCC-BY-NC-ND 4.0 International license.

A diameter and length (acoustic on/off)

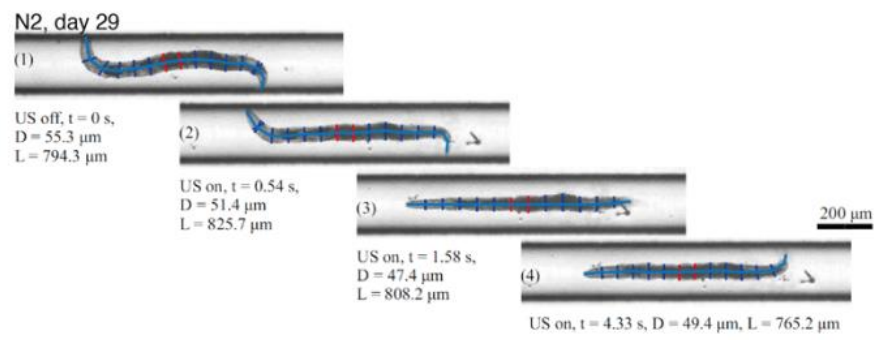

\section{B Assumptions \& simplifications}

- Rod shaped intestine

- No volume variation during measurement

- No density and compressibility changes

- "Sphere" assumption for acoustic force calculation

- Energy calculation includes no turns folds since these periods are inaccessible to image processing

- Batchwise measurement along the lifespan and the batches are assumed to be identical.
C Intestine length, diameter, pharynx size and wrinkles

- Wrinkles occur rarely and only at very high age ( $>25$ days)

- Intestine and pharynx are often difficult to detect

- Quantification ability is dependent on the rotation of the animal

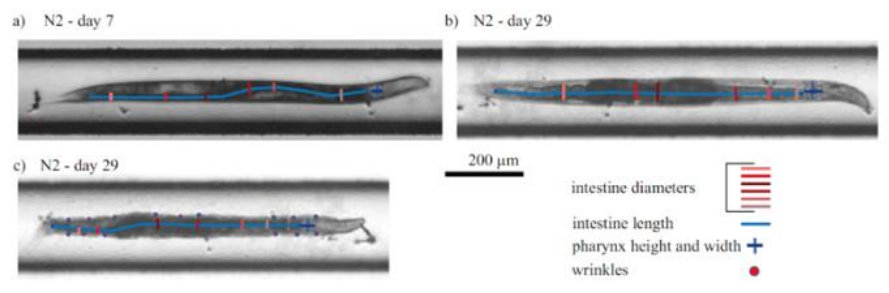

E Intensity heterogeneity across sections

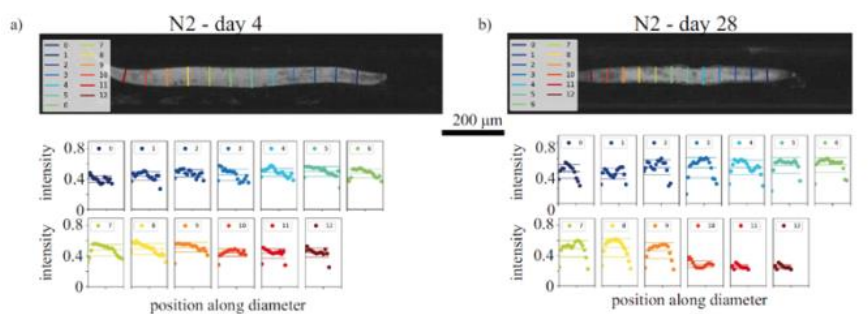

D Phenotype interdependence

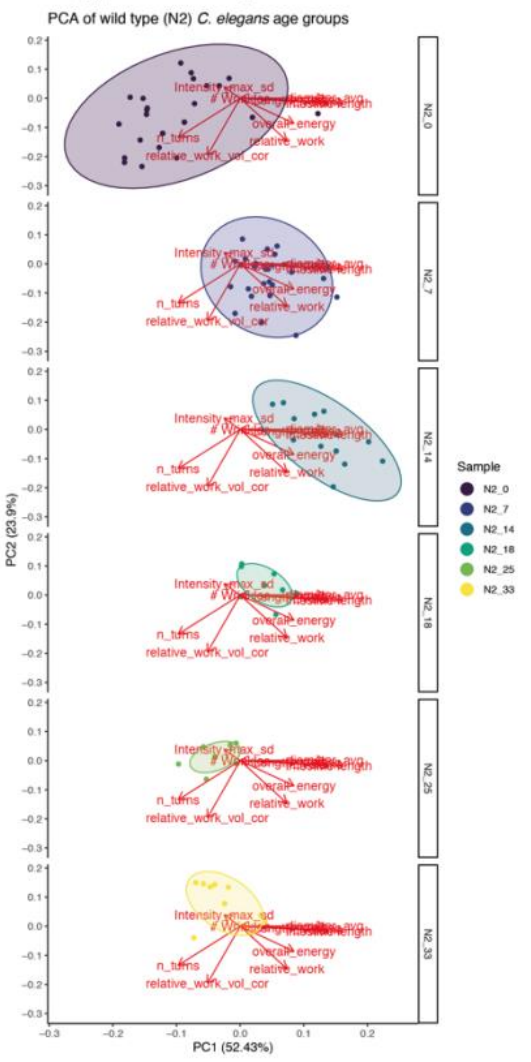

F Increasing bending angles with age

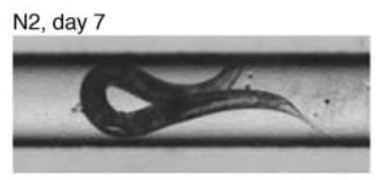

N2, day 28

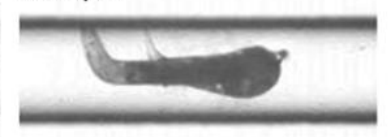

G Shrinking and wrinkle occurence with age

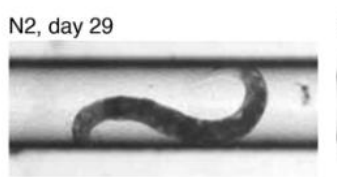

N2, day 29

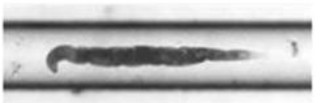

\section{H Acoustic field triggers escape response}
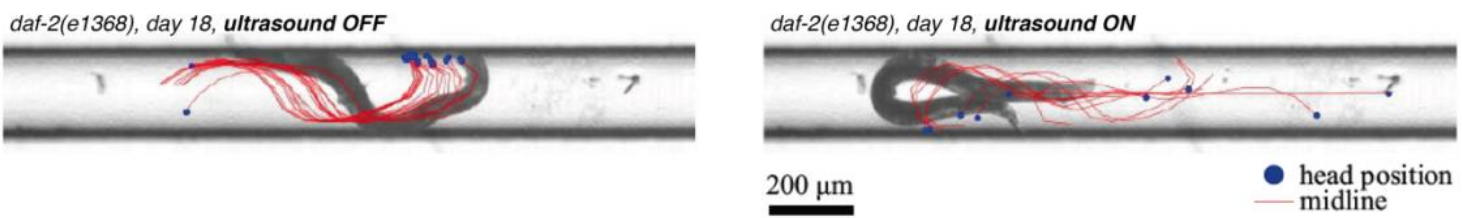

Extended Data Fig. 4. Phenotypes quantified within the microfluidic setup:

611 Automated quantification of $C$. elegans diameter $(D=$ diameter $)$ evaluated at the middle segments colored in red and the

612 length $(L=$ length $)$ of the animal along its spine colored in light blue $(A)$, The change in diameter and length quantification as the field is switched (US = ultrasound) is indicated. The assumptions and necessary simplifications that were made to analyze the acquired frames are listed $(B)$. Excerpts of the manual quantification of $C$. elegans intestine length and diameter, pharynx width and height, and the number of cuticle wrinkles are displayed $(C)$. Annotation was performed in a selfdeveloped image annotator suite based on the python programming language. Principal component analysis (PCA) of the 
bioRxiv preprint doi: https://doi.org/10.1101/2021.05.31.446397; this version posted May 31, 2021. The copyright holder for this preprint

(which was not certified by peer review) is the author/funder, who has granted bioRxiv a license to display the preprint in perpetuity. It is made available under aCC-BY-NC-ND 4.0 International license.

617

618

619

620

621

622

623

624

625

626

measured phenotypes are displayed for aging wild type (N2) C. elegans individuals, with each point representing one individual. The overlaying components refer to the highly correlated features length, volume, and diameter. Tissue heterogeneity and age pigments were studied by analyzing the intensity distribution across different cross-sections along the animal's fitted spine. Representative images of tissue and cuticle weakening displaying an increased bending angle of older animals compared to younger individuals as they attempt to turn in the channel $(F)$. Similarly, older animals are smaller and sometimes display cuticle wrinkles $(G)$. The strong stimulation of the acoustic force in the animals is shown for a representative daf-2(e1368) animal $(H)$. The spline midline and head position is displayed for seconds preceding (left), and after (right), the acoustic field is activated. The animal directly responds with a strong escape response. 
bioRxiv preprint doi: https://doi.org/10.1101/2021.05.31.446397; this version posted May 31, 2021. The copyright holder for this preprint (which was not certified by peer review) is the author/funder, who has granted bioRxiv a license to display the preprint in perpetuity. It is made available under aCC-BY-NC-ND 4.0 International license.

\section{Scaled \& unscaled phenotypes}

A
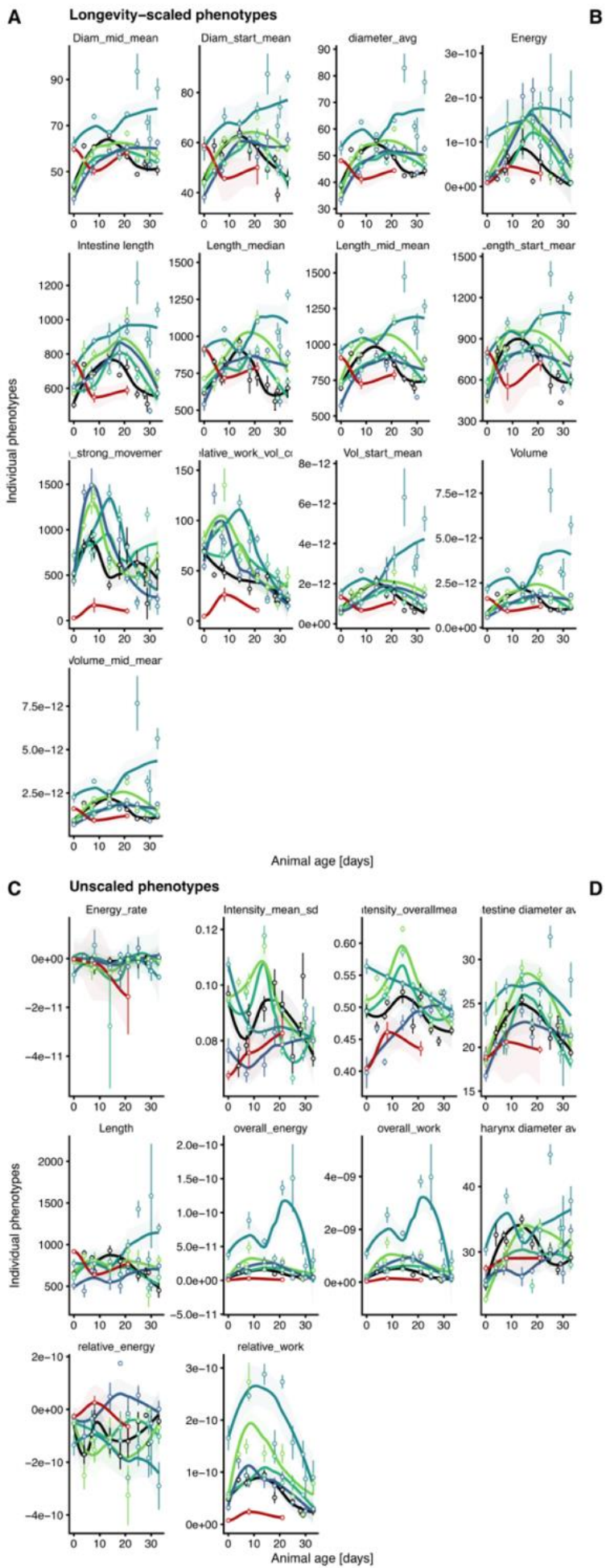

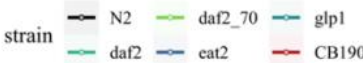

B
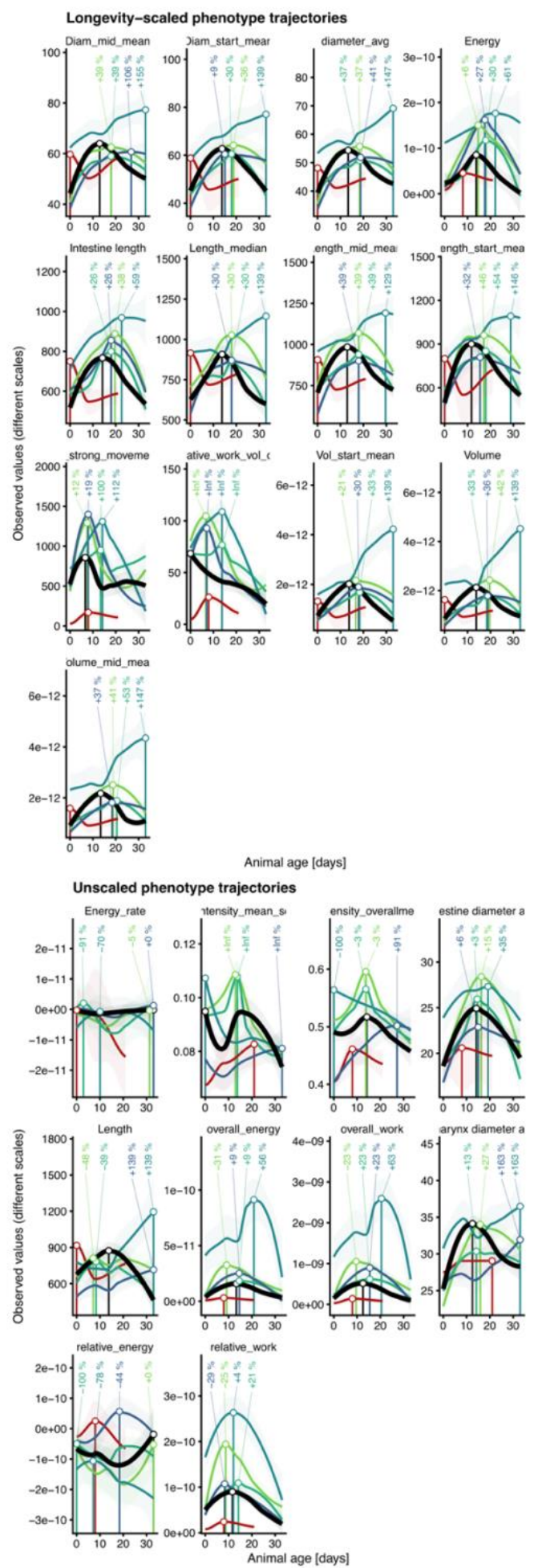

Extended Data Fig. 5: Rationale behind the partitioning of physical properties into scaled and unscaled phenotypes.

Phenotypes displaying an age-dependent change and at least a partial temporal shift for any long-lived strain were manually classified as temporally scaled phenotypes (A, $B$ ), and phenotypes that do not satisfy these conditions are categorized as unscaled phenotypes $(C, D)$. The quantified phenotypes are fitted using a loess model in all panels. The observed values for each individual are displayed using mean $+/$ - standard error for each timepoint $(A, C)$. The maximum value predicted by the 
bioRxiv preprint doi: https://doi.org/10.1101/2021.05.31.446397; this version posted May 31, 2021. The copyright holder for this preprint (which was not certified by peer review) is the author/funder, who has granted bioRxiv a license to display the preprint in perpetuity. It is made available under aCC-BY-NC-ND 4.0 International license.

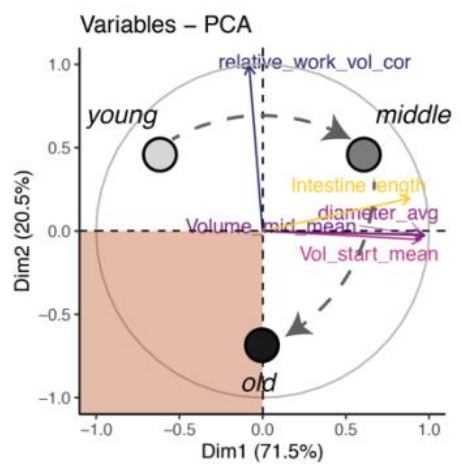

B PCA of $C$. elegans genotypes and age groups

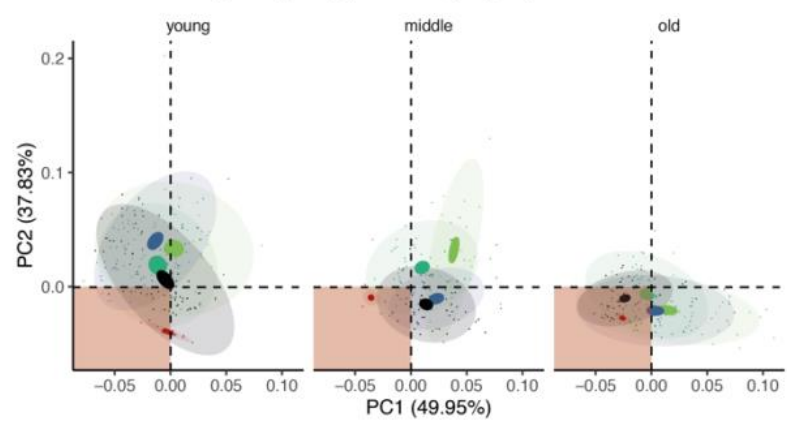

C PCA of $C$. elegans genotypes and age groups

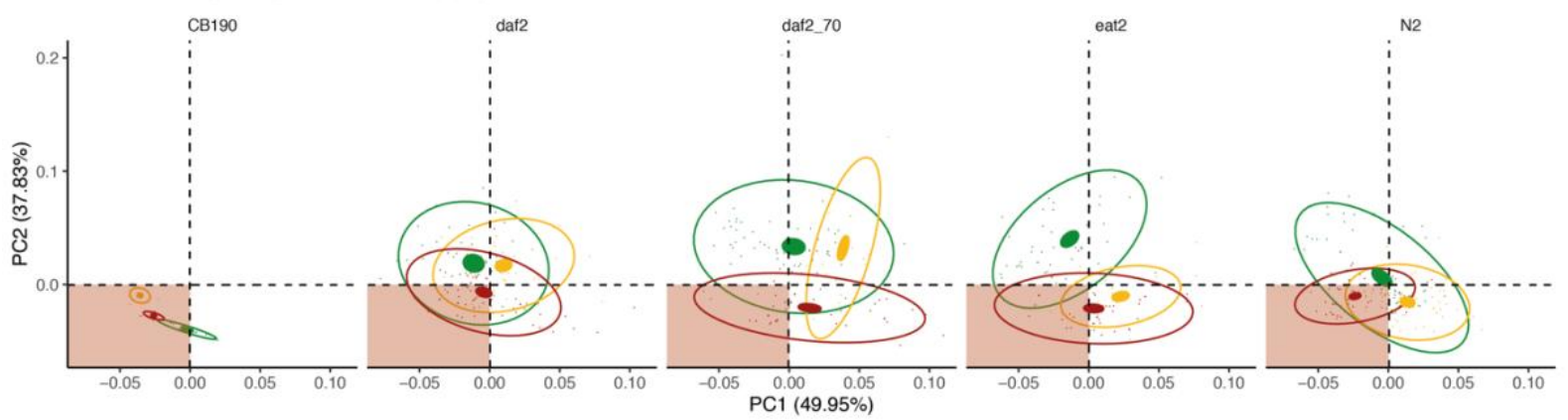

D PCA of wild type (N2) C. elegans age groups

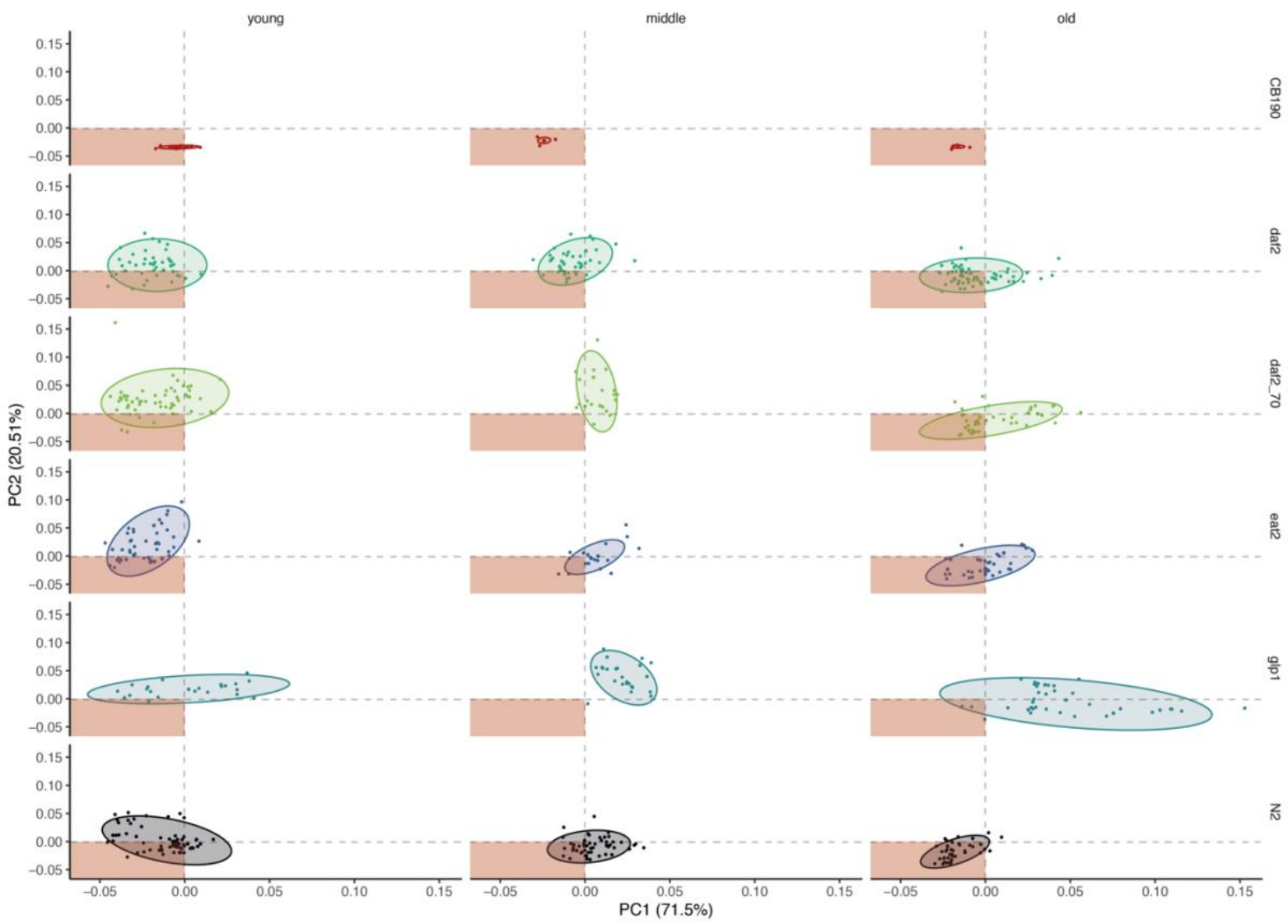

Extended Data Fig. 6: Principal component analysis of temporally scaled phenotypes to identify similarity between phenotypes and their contributions.

The subset of temporally scaled phenotypes for each individual was subjected to principal component analysis, and the first two principle components are shown together with the variance they explain. The contribution of each phenotype to the two first principle components is displayed as vectors with the orientation reflecting the directionality and the color and length capturing the contribution of each phenotype $(A)$. The overall progression of young to middle-aged to old individuals through the phenotype landscape is schematically illustrated. The bottom left quadrant is highlighted in red to indicate its association with animals experience a poor health status. To compare the effect of age on the clustering of the different $C$. elegans genotypes all animals were grouped into 3 age categories, and the individual genotypes were shown in different 
bioRxiv preprint doi: https://doi.org/10.1101/2021.05.31.446397; this version posted May 31, 2021. The copyright holder for this preprint

(which was not certified by peer review) is the author/funder, who has granted bioRxiv a license to display the preprint in perpetuity. It is made available under aCC-BY-NC-ND 4.0 International license.

646

647

648

649

colors (B). Confidence ellipses are drawn at the level of $95 \%$ for all samples (transparent) and the sample means (nontransparent). To compare the effect of age separately for each genotype, the three age categories are shown for each strain, young in green, middle-aged in yellow and old animals in red $(C)$. Complete separation of age and genotype is provided in panel (D), highlighting the measurements for every individual animal encompassed by the $95 \%$ sample confidence ellipse. 
bioRxiv preprint doi: https://doi.org/10.1101/2021.05.31.446397; this version posted May 31,2021 . The copyright holder for this preprint

(which was not certified by peer review) is the author/funder, who has granted bioRxiv a license to display the preprint in perpetuity. It is made available under aCC-BY-NC-ND 4.0 International license.

A N2, daf2, daf2_70, eat2, glp1

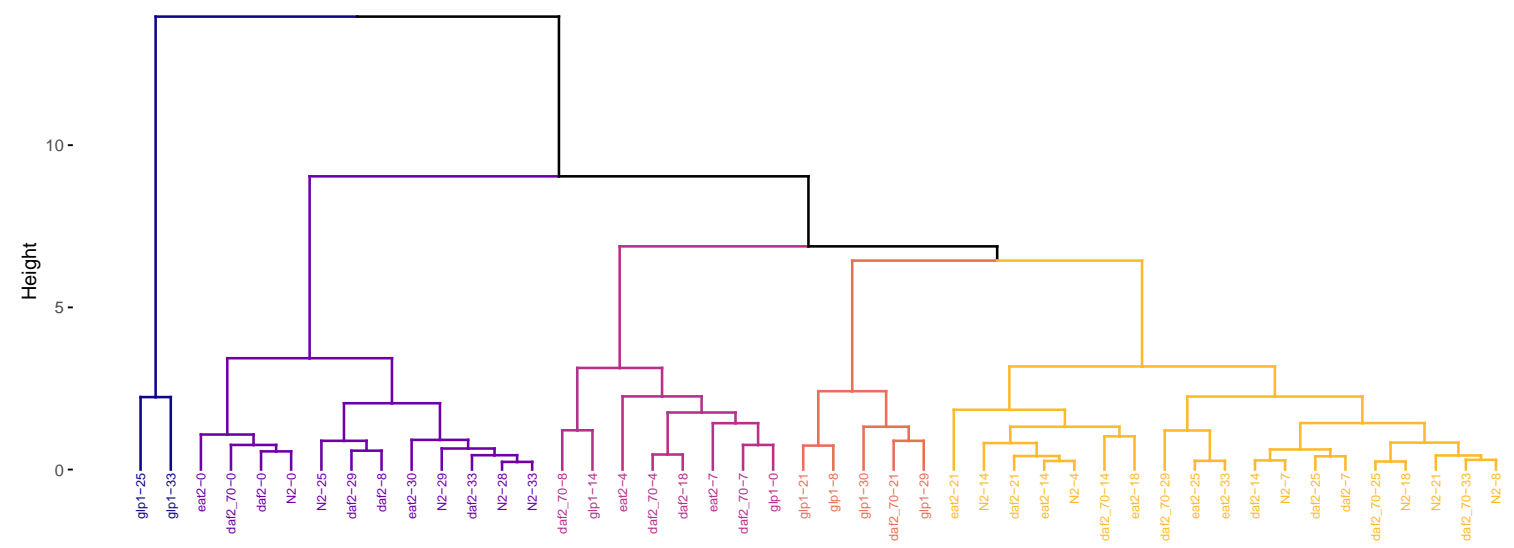

B $\quad$ N2, eat2

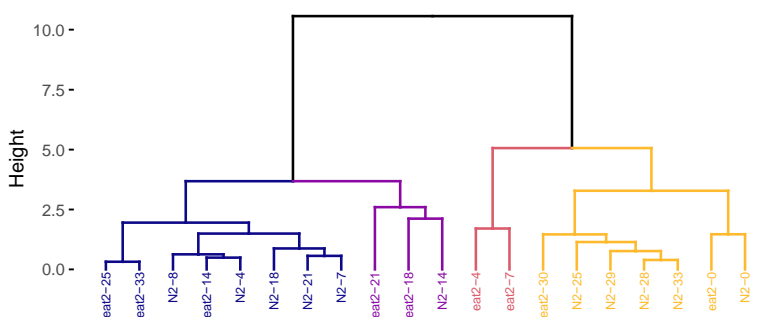

D $\quad \mathrm{N} 2$, glp 1

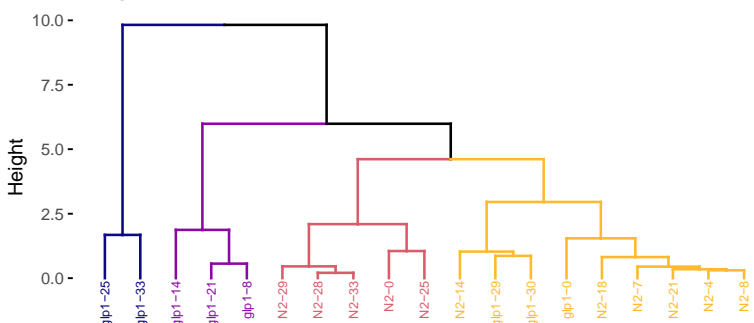

F $\quad$ N2, daf2

10.0 -

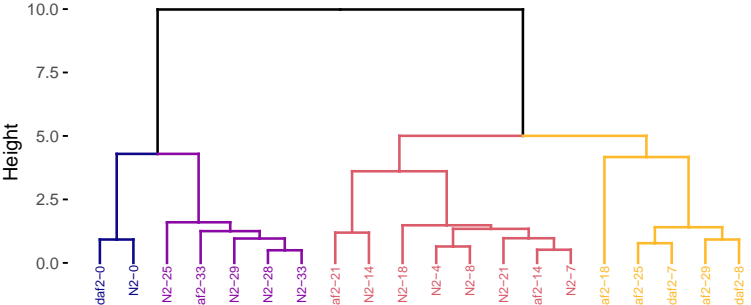

H

N2, daf2_70

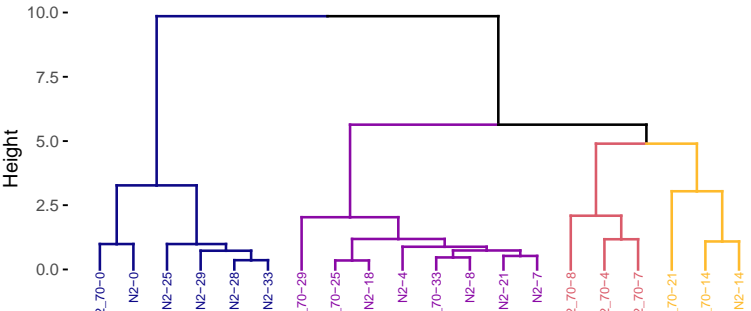

C eat2

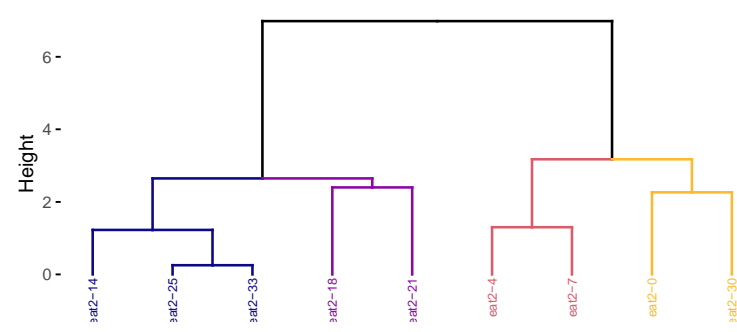

E glp1

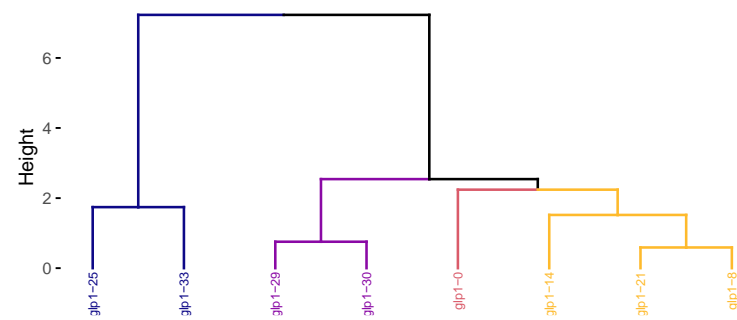

G daf2

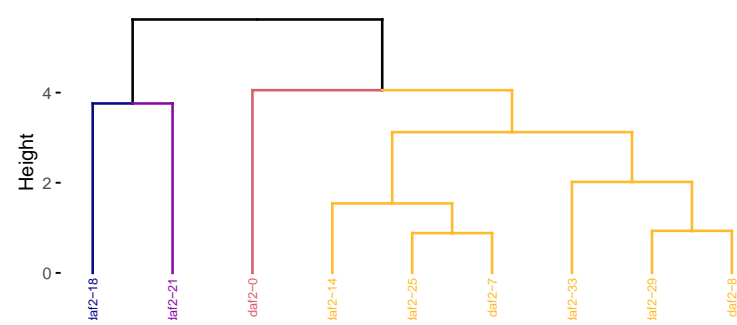

I daf2 70

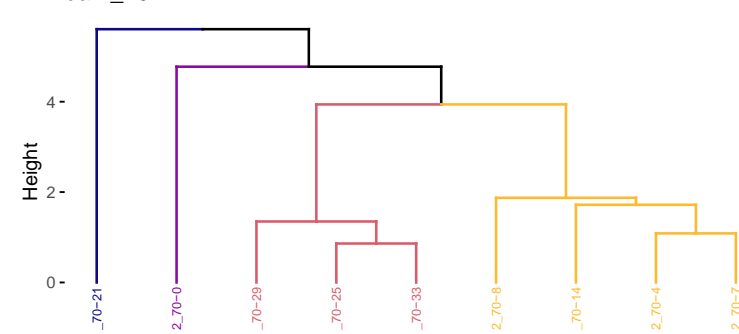

Extended Data Fig.7: Hierarchical clustering of temporally scaled phenotypes.

The similarity between sampled genotypes and ages are displayed using hierarchical clustering. The comparison between all samples $(A)$, between each long-lived genotype and wild type $(B, D, F, H)$, and within each long-lived is shown $(C, E, G, I)$. The tree is cut in five clusters when comparing all samples and in all other cases in four clusters and colored from left to 
bioRxiv preprint doi: https://doi.org/10.1101/2021.05.31.446397; this version posted May 31,2021 . The copyright holder for this preprint (which was not certified by peer review) is the author/funder, who has granted bioRxiv a license to display the preprint in perpetuity. It is made available under aCC-BY-NC-ND 4.0 International license.
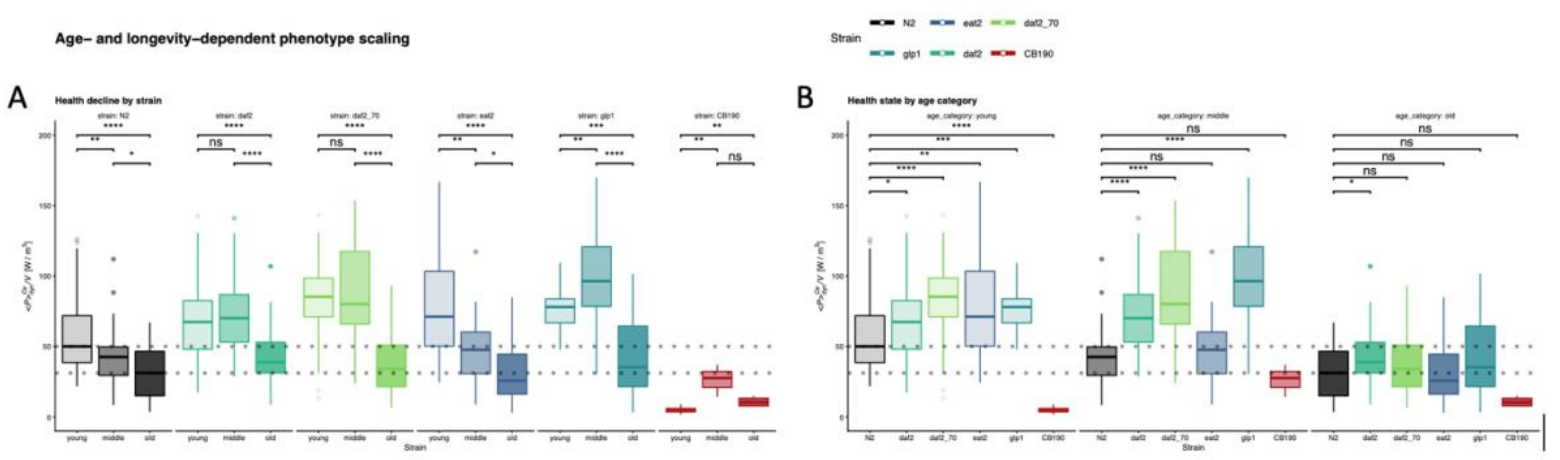

\section{Combined healthspan metrics}
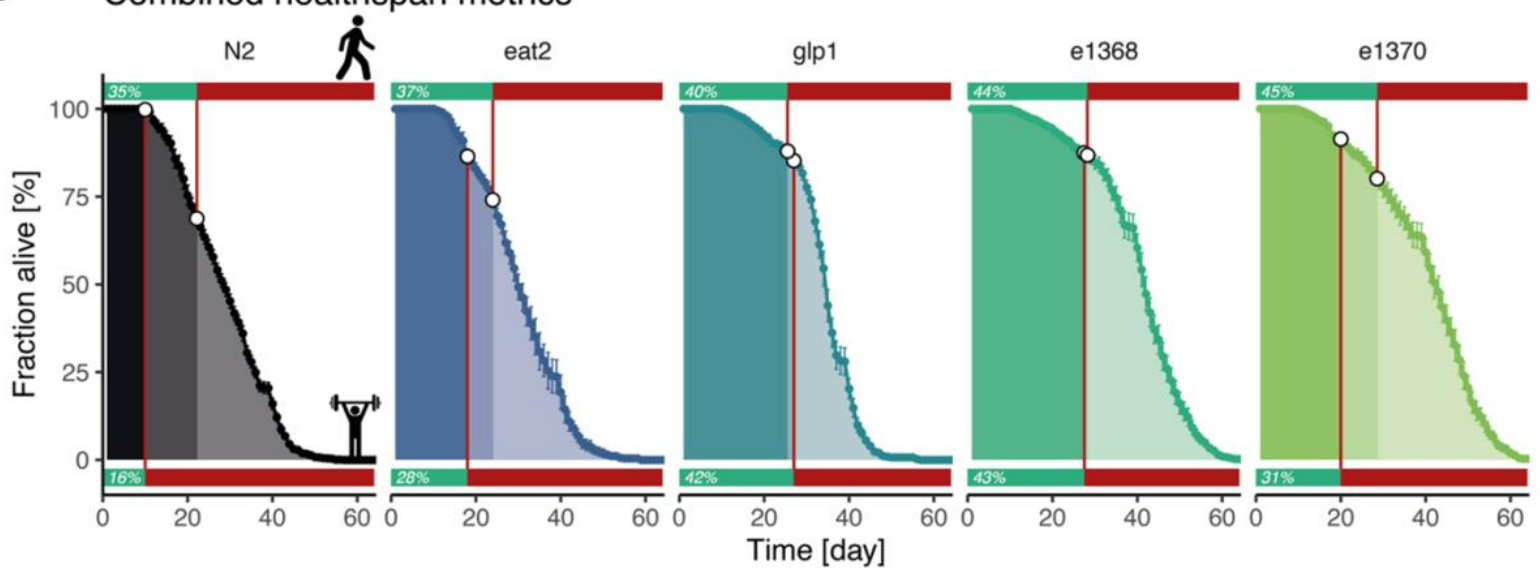

Figure 6 Integration of voluntary movement and forced maximum muscle strength quantification to yield a comprehensive understanding of $\mathbf{C}$. elegans healthspan.

The volume-corrected relative work performed by each genotype and grouped by age category (young $<=7$ days, $8<$ middle $<20$ days, old $>=20$ days) is shown as boxplots facetted by genotype $(A)$ and by age category $(B)$. P-values of selected comparisons (Mann-Whitney test) are displayed as symbols $(n s>0.05, *<0.05, * *<0.01, * * *<0.001, * * * *<0.0001)$. The Population medians for young and old N2 are displayed as horizontal dashed lines across all panels.

$C)$ The measured lifespan of each C. elegans genotype is displayed with animal age on the $x$-axis, the fraction of the population that is alive on the $y$-axis, and the mean and standard error between plates shown as point and line range. The voluntary movement was quantified using the active vs sedentary behavior of the unstimulated animals on plates and is shown for each population as a bar at the top of each panel. Muscle health and the corresponding onset of sickspan due to reduced muscle function is depicted as a bar at the bottom of each facet. The position on the lifespan curve corresponding to the health-to-sickspan transition of either the unstimulated or stimulated healthspan quantification is marked by a white circle. The two health assessments divide the lifespan curve into three segments with decreasing health status reflected by increasing transparency. 
693

694

695

696

697

698

699

700

701

702

703

704

705

706

707

708

709

710

711

712

713

714

715

716

717

\section{Materials and Methods}

\section{Strains}

Caenorhabditis elegans strains were maintained on NGM plates and OP50 Escherichia coli bacteria. The wild-type strain was N2 Bristol. Mutant strains used are described at www.wormbase.org: LGII: eat-2(ad1116); LGIII: daf-2(e1368, e1370), glp-1(e2141).

\section{C. elegans culturing conditions}

C. elegans populations were age-synchronized by isolating eggs from gravid C. elegans adults, incubating them for 18 hours in M9 until hatching in the presence of $5 \mu \mathrm{g} \mathrm{mL}-1$ cholesterol (Sigma-Aldrich). Hatched L1-larvae were grown on standard culturing NGM OP50 plates and then shifted to $50 \mathrm{mM}$ FUDR plates seeded with heat-killed OP50 plates when reaching the L4 state. Animals were maintained on FUDR plates until the measurement of different ages was taken. The $g l p-1$ genotype was placed at $25^{\circ} \mathrm{C}$ after bleach treatment and shifted back to $15^{\circ} \mathrm{C}$ at the L4 stage and otherwise treated equally to the other strains. Except for $g l p-1$ during its development, all animals were always maintained and aged at $15^{\circ} \mathrm{C}$.

\section{Automated survival assays using the lifespan machine}

To compare the lifespans among wild type and long-lived mutants, we raised all animals for several generations in parallel. Automated survival analysis was conducted using the lifespan machine setup described by ${ }^{26}$. Briefly, approximately 1000 L4 animals were resuspended in M9 and transferred to NGM plates containing $50 \mu \mathrm{M}$ 5-Fluoro-2'deoxyuridine (FUdR) seeded with heat-killed OP50 bacteria and incubated at $15^{\circ} \mathrm{C}$ until day 4 of adulthood. Animals were then resuspended in M9 and transferred to fresh FUDR plates containing tight-fitting lids (BD Falcon Petri Dishes, 50x9mm), and the plates were dried with their lids open for 30 minutes after transfer. The plates were incubated for five additional days to rule out contamination and then loaded in the lifespan machine. Air-cooled Epson V800 scanners were utilized for all 
experiments operating at a scanning frequency of one scan of 30 minutes. Temperature probes

720 (Thermoworks, Utah, US) were used to monitor the temperature on the scanner flatbed and kept constant at $15^{\circ} \mathrm{C}$.

722

Voluntary movement healthspan measured by lifespan machine

724 The time point at which the animal stops moving completely and irretrievably is classified as the point of death and defines the lifespan of each individual. The health- to sickspan transition is estimated by the time point when major movement ceases and exclusively head movements, posture change, and minor body movements can be observed. The animal is also required to be sedentary and remains in the rough vicinity of the area; it will ultimately die.

\section{Microfluidics device measuring muscle strength}

The detailed description of the microfluidics device development and characterization is found in the Supplementary Methods file.

\section{Oxidative stress assay arsenite with automated wormtracker}

Voluntary movement in liquid, as well as resistance to oxidative stress, was quantified using the wMicroTracker (NemaMetrix) platform. Briefly, animals were resuspended in M9 at

742 deviation of wells, are shown. 
745 The analysis was performed using the statistical software R. data processing and visualization

746 was performed using the tidyverse package collection, most prominently dplyr and ggplot2.

747 Furthermore, packages were used for lifespan analysis (survival, survminer), computing and

748 visualizing PCA (stats and ggfortify, factoextra), fitting loess models (stats), and segmented fits

749 (segmented), labeling (ggrepel) comparing distributions (ggpubr) and arranging figures 750 (cowplot).

751

752 


\section{References}

754

755

756

757

758

759

760

761

762

763

764

765

766

767

768

769

770

771

772

773

774

775

776

777

778

779

780

781

782

783

784

1. Nations, D. of E. and S. A. P. D. U. World Population Ageing 2019: Highlights. 1-46 (2019).

2. Partridge, L., Deelen, J. \& Slagboom, P. E. Facing up to the global challenges of ageing. Nature 561, 45-56 (2018).

3. Goldman, D. P. et al. Substantial health and economic returns from delayed aging may warrant a new focus for medical research. Health affairs (Project Hope) 32, 1698-1705 (2013).

4. Kaeberlein, M. Translational geroscience: A new paradigm for 21st century medicine. Translational Medicine of Aging 1, 1-4 (2017).

5. Kennedy, B. K. et al. Geroscience: linking aging to chronic disease. Cell 159, 709-713 (2014).

6. Olshansky, S. J. From Lifespan to Healthspan. JAMA : the journal of the American Medical Association 320, 1323-1324 (2018).

7. Ailshire, J. A., Beltrán-Sánchez, H. \& Crimmins, E. M. Becoming centenarians: disease and functioning trajectories of older US Adults as they survive to 100. The journals of gerontology Series A, Biological sciences and medical sciences 70, 193-201 (2015).

8. Andersen, S. L., Sebastiani, P., Dworkis, D. A., Feldman, L. \& Perls, T. T. Health span approximates life span among many supercentenarians: compression of morbidity at the approximate limit of life span. The journals of gerontology Series A, Biological sciences and medical sciences 67, 395-405 (2012).

9. Evans, C. J. et al. Place and cause of death in centenarians: a population-based observational study in England, 2001 to 2010. PLoS medicine 11, e1001653 (2014).

10. Evert, J., Lawler, E., Bogan, H. \& Perls, T. Morbidity profiles of centenarians: survivors, delayers, and escapers. The Journals of Gerontology: Series A 58, 232-237 (2003).

11. Ismail, K. et al. Compression of Morbidity Is Observed Across Cohorts with Exceptional Longevity. Journal of the American Geriatrics Society 64, 1583-1591 (2016).

12. Kheirbek, R. E. et al. Characteristics and Incidence of Chronic Illness in CommunityDwelling Predominantly Male U.S. Veteran Centenarians. Journal of the American Geriatrics Society 65, 2100-2106 (2017).

13. Kenyon, C. J. The genetics of ageing. Nature 464, 504-512 (2010).

14. López-Otín, C., Blasco, M. A., Partridge, L., Serrano, M. \& Kroemer, G. The hallmarks of aging. Cell 153, 1194-1217 (2013). 
17. Hahm, J.-H. et al. C. elegans maximum velocity correlates with healthspan and is maintained in worms with an insulin receptor mutation. Nature communications $\mathbf{6}, 8919$ 795 (2015).

796

797

798

799

800

801

802

803

804

805

806

807

808

809

810

811

812

813

814

815

816

817

818

819

820

18. Huang, C., Xiong, C. \& Kornfeld, K. Measurements of age-related changes of physiological processes that predict lifespan of Caenorhabditis elegans. Proceedings of the National Academy of Sciences of the United States of America 101, 8084-8089 (2004).

19. Stamper, B. L. N. et al. Movement decline across lifespan of Caenorhabditis elegans mutants in the insulin/insulin-like signaling pathway. Aging cell 17, e12704 (2018).

20. Podshivalova, K. \& Kerr, R. A. How a Mutation that Slows Aging Can Also Disproportionately Extend End-of-Life Decrepitude. Cell reports 19, 441-450 (2017).

21. Ewald, C. Y., Landis, J. N., Abate, J. P., Murphy, C. T. \& Blackwell, T. K. Dauerindependent insulin/IGF-1-signalling implicates collagen remodelling in longevity. Nature 519, 97-101 (2015).

22. Ewald, C. Y., Castillo-Quan, J. I. \& Blackwell, T. K. Untangling Longevity, Dauer, and Healthspan in Caenorhabditis elegans Insulin/IGF-1-Signalling. Gerontology 64, 96-104 (2018).

23. Gems, D. et al. Two pleiotropic classes of daf-2 mutation affect larval arrest, adult behavior, reproduction and longevity in Caenorhabditis elegans. Genetics 150, 129-155 (1998).

24. Hess, M., Gomariz, A., Goksel, O. \& Ewald, C. Y. In-vivo quantitative image analysis of age-related morphological changes of $\mathrm{C}$. elegans neurons reveals a correlation between neurite bending and novel neurite outgrowths. Eneuro 6, ENEURO.0014-19.2019 (2019).

25. Leong, D. P. et al. Prognostic value of grip strength: findings from the Prospective Urban Rural Epidemiology (PURE) study. Lancet 386, 266-273 (2015).

26. Stroustrup, N. et al. The Caenorhabditis elegans Lifespan Machine. Nature Methods 10, 665-670 (2013).

27. Zhang, W. B. et al. Extended Twilight among Isogenic C. elegans Causes a

Disproportionate Scaling between Lifespan and Health. Cell Systems 3, 333-345.e4 (2016).

28. Petrella, J. K., Kim, J., Tuggle, S. C., Hall, S. R. \& Bamman, M. M. Age differences in knee extension power, contractile velocity, and fatigability. J Appl Physiol 98, 211-220 (2005). 
824 29. Kostka, T. Quadriceps maximal power and optimal shortening velocity in 335 men aged

825 23-88 years. Eur J Appl Physiol 95, 140-145 (2005).

826 30. Baasch, T. et al. Acoustic Compressibility of Caenorhabditis elegans. Biophysical journal

827 115, 1817-1825 (2018).

828 31. Donnelly, J. L. et al. Monoaminergic Orchestration of Motor Programs in a Complex C.

829 elegans Behavior. Plos Biol 11, e1001529 (2013).

830 32. Hulme, S. E. et al. Lifespan-on-a-chip: microfluidic chambers for performing lifelong

831 observation of C. elegans. Lab on a chip 10, 589-597 (2010).

832 33. Shi, C. \& Murphy, C. T. Mating induces shrinking and death in Caenorhabditis mothers.

833 Science (New York, NY) 343, 536-540 (2014).

834 34. Ezcurra, M. et al. C. elegans Eats Its Own Intestine to Make Yolk Leading to Multiple

835 Senescent Pathologies. Current biology: CB 28, 2544-2556.e5 (2018).

836 35. Gilpin, W., Uppaluri, S. \& Brangwynne, C. P. Worms under Pressure: Bulk Mechanical

837 Properties of C. elegans Are Independent of the Cuticle. Biophysical journal 108, 1887-1898

838 (2015).

839 36. Essmann, C. L. et al. Mechanical properties measured by atomic force microscopy define

840 health biomarkers in ageing C. elegans. Nature communications 11, 1043 (2020).

841 37. Olshansky, S. J. \& Carnes, B. A. Inconvenient Truths About Human Longevity. The

842 journals of gerontology Series A, Biological sciences and medical sciences 74, S7-S12

843 (2019).

844 38. Freund, A. Untangling Aging Using Dynamic, Organism-Level Phenotypic Networks.

845 Cell Systems 8, 172-181 (2019).

846 39. Gems, D. The aging-disease false dichotomy: understanding senescence as pathology.

847 Frontiers in genetics 6, 212 (2015).

848 40. Ayyadevara, S., Alla, R., Thaden, J. J. \& Reis, R. J. S. Remarkable longevity and stress

849 resistance of nematode PI3K-null mutants. Aging cell 7, 13-22 (2008).

850 41. Stroustrup, N. et al. The temporal scaling of Caenorhabditis elegans ageing. Nature 530,

$851 \quad 103-107$ (2016).

852 42. Tarkhov, A. E. et al. A universal transcriptomic signature of age reveals the temporal

853 scaling of Caenorhabditis elegans aging trajectories. Scientific reports 9, 7368 (2019).

854 43. Gladyshev, V. N. The origin of aging: imperfectness-driven non-random damage defines 855 the aging process and control of lifespan. Trends in genetics : TIG 29, 506-512 (2013).

856 44. Lippuner, A. D., Julou, T. \& Barral, Y. Budding yeast as a model organism to study the 857 effects of age. FEMS microbiology reviews 38, 300-325 (2014). 
858 45. Yang, Y. et al. Temporal scaling of aging as an adaptive strategy of Escherichia coli.

859 Science advances 5, eaaw2069 (2019).

860 46. Kuo, P. L. et al. A roadmap to build a phenotypic metric of ageing: insights from the

861 Baltimore Longitudinal Study of Aging. Journal of Internal Medicine 287, 373-394 (2020).

862 47. Ash, A. S. et al. Are Members of Long-Lived Families Healthier Than Their Equally

863 Long-Lived Peers? Evidence From the Long Life Family Study. The journals of gerontology

864 Series A, Biological sciences and medical sciences 70, 971-976 (2015).

865 48. Sebastiani, P. et al. Families Enriched for Exceptional Longevity also have Increased

866 Health-Span: Findings from the Long Life Family Study. Frontiers in public health 1, 38

867 (2013).

868 49. Freedman, V. A., Wolf, D. A. \& Spillman, B. C. Disability-Free Life Expectancy Over 30

869 Years: A Growing Female Disadvantage in the US Population. American journal of public

870 health 106, 1079-1085 (2016).

871 50. Fischer, K. E. et al. A cross-sectional study of male and female C57BL/6Nia mice

872 suggests lifespan and healthspan are not necessarily correlated. Aging 8, 2370-2391 (2016).

873 51. Garcia-Valles, R. et al. Life-long spontaneous exercise does not prolong lifespan but

874 improves health span in mice. Longevity \& healthspan 2, 14 (2013).

875 52. Mattison, J. A. et al. Impact of caloric restriction on health and survival in rhesus

876 monkeys from the NIA study. Nature 489, 318-321 (2012).

877 53. Bellantuono, I. et al. A toolbox for the longitudinal assessment of healthspan in aging 878 mice. Nature Protocols 15, 540-574 (2020).

879 54. Collins, J. J., Huang, C., Hughes, S. \& Kornfeld, K. The measurement and analysis of 880 age-related changes in Caenorhabditis elegans. WormBook: the online review of C elegans 881 biology 1-21 (2008) doi:10.1895/wormbook.1.137.1.

882 55. Teuscher, A. C., Statzer, C., Pantasis, S., Bordoli, M. R. \& Ewald, C. Y. Assessing 883 Collagen Deposition During Aging in Mammalian Tissue and in Caenorhabditis elegans. 884 Methods Mol Biology Clifton N J 1944, 169-188 (2019).

885 56. Haefke, A. \& Ewald, C. Tail Tendon Break Time for the Assessment of Aging and 886 Longitudinal Healthspan in Mice. Bio-protocol 10, (2020).

887 57. Teuscher, A. C. \& Ewald, C. Y. Overcoming Autofluorescence to Assess GFP Expression 888 During Normal Physiology and Aging in Caenorhabditis elegans. BIO-PROTOCOL 8, (2018). 